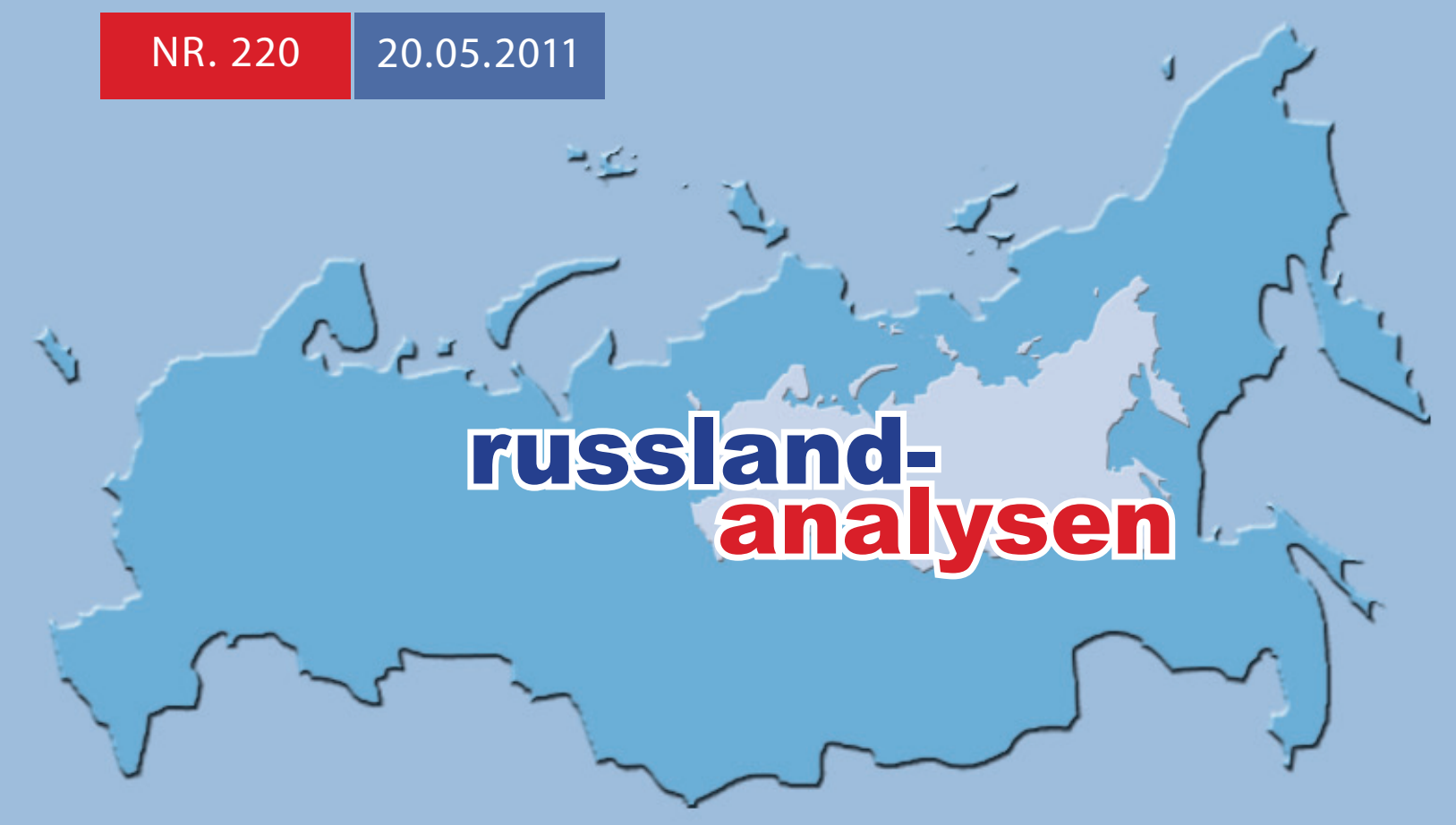

http://www.laender-analysen.de/russland/

\title{
TERRORISMUSBEKÄMPFUNG IN RUSSLAND
}

aNALYSE

Terrorismus in Russland:

von der existenziellen Bedrohung zum Sicherheitsrisiko und einer konzeptionellen Sackgasse

Aglaya Snetkov, Zürich

ANALYSE

Asymmetrien der Terrorismusbekämpfung

Michail Logvinov, Chemnitz

CHRONIK

Bürgerkriegsaktionen und Terrorakte in Russland seit Dezember 2010

- UMFRAGE

Terrorismus und Terrorismusbekämpfung in der russischen öffentlichen Meinung

CHRONIK

Vom 5. bis zum 19. Mai 2011

- LESETIPP

Abstracts aus Osteuropa 2-3/2011 »Logbuch Arktis»

\section{LESETIPP}

Abstracts aus Osteuropa 4/2011 mit Russlandbezug

\section{LESETIPP}

Neu: Belarus-Analysen 


\section{Terrorismus in Russland: von der existenziellen Bedrohung zum Sicherheitsrisiko und einer konzeptionellen Sackgasse}

Aglaya Snetkov, Zürich

\section{Zusammenfassung}

Russlands Krieg gegen den Terrorismus dauert nun schon über einem Jahrzehnt. Wie der Terroranschlag vom 24. Januar 2011 auf dem Flughafen Domodedowo gezeigt hat, ist die Gefahr weiterer Anschläge jedoch nicht so schnell gebannt. Der vorliegende Beitrag stellt Russlands Umgang mit dem Terrorismus seit 1999 dar und vertritt die Position, dass die Terrorgefahr, die anfangs als eine existenzielle Bed rohung für den russischen Staat und die Nation dargestellt wurde, in den letzten Jahren zu einem bloßen Sicherheitsrisiko herabgestuft wurde. Da sich die russische Führung bezüglich der Ausrichtung ihrer Strategie zur Terrorismusbekämpfung gegenwärtig allem Anschein nach in einer konzeptionellen Sackgasse befindet, schließt die Autorin mit einer pessimistischen Prognose. Russland wird das Terrorproblem in den nächsten fünf Jahren nicht erfolgreich bewältigen können.

\section{Domodedowo und die Kontextualisierung des Krieges gegen den Terror}

Am 24. Januar 2011 wurde Moskau durch einen Selbstmordanschlag im Ankunftsbereich des internationalen Terminals von Russlands größtem Flughafen Domodedowo erschüttert. 37 Menschen kamen ums Leben, Dutzende wurden verletzt. Dieser jüngste Anschlag reiht sich ein in eine Serie von Terroranschlägen, die in den vergangenen Jahren auf die Transportinfrastruktur und das Zentrum Russland verübt wurden, mit der kleinen Veränderung, dass dieses Mal allem Anschein nach ausländische Staatsbürger zur Zielgruppe erkoren wurden.

Experten haben in den vergangenen Monaten versucht, sich einen Reim auf den Anschlag zu machen. Bei der Analyse des Anschlags haben sie eine Reihe von Fehlern des russischen Staates und seiner Politik im Nordkaukasus benannt und versucht, den Charakter der gegenwärtigen terroristischen Bedrohung innerhalb Russlands zu identifizieren. Mehrere unterschiedliche Maßnahmen zur Vorbeugung weiterer Anschläge wurden vorgeschlagen, darunter die Notwendigkeit, eine effizientere Strategie zum Kampf gegen den Terrorismus zu entwickeln, zu einer fundierteren sozioökonomischen Politik im Nordkaukasus überzugehen, das politische Systems im ganzen Land zu reformieren und die Korruption zu beseitigen sowie sich im Rahmen der Bemühungen, die lokale Bevölkerung in der Region zu befrieden, stärker auf die "Gewinnung von Herzen und Köpfen« im Nordkaukasus zu konzentrieren.

Im Kern argumentieren die meisten Kommentatoren, dass Russland die Bedrohung durch den Terrorismus erst dann angehen kann, wenn es die größeren strukturellen, aber auch Führungsprobleme überwindet, die bis heute dafür verantwortlich sind, dass Russland eine schwache Macht ist, die mit einer wachsenden Terrorgefahr auf ihrem Staatsgebiet konfrontiert ist.
Wie es scheint, leidet Russland sowohl unter einem konzeptionellen Scheitern, da es keine umfassende Strategie im Umgang mit der terroristischen Bedrohung auf seinem Staatsgebiet entwickeln hat, wie auch darunter, dass Kapazitäten fehlen, eine solche Strategie effektiv umzusetzen. Dieser Beitrag untersucht das konzeptionelle Scheitern des russischen Führungspersonals in Bezug auf den Terrorismus. Hierzu werden die Positionen im Umgang mit dem Terrorismus seit dem Ausbruch des zweiten Tschetschenienkriegs 1999 analysiert.

\section{Terrorismus in Russland}

Der Terroranschlag von Domodedowo war kein einmaliger Vorfall, sondern steht in einer langen Anschlagsreihe, die Russland in den vergangen zehn Jahren erschüttert hat. In den letzten Jahren ereigneten sich einige Anschläge, darunter zwei Selbstmordattentate in der Moskauer U-Bahn am 29. März 2010, die 40 Personen töteten und 100 weitere verletzten; die Anschläge auf den Hochgeschwindigkeitszug Moskau-St. Petersburg am 27. November 2009 und am 13. August 2007 sowie ein Anschlag auf einen Bus am 31. August 2007 in Togliatti, Südrussland. Diese Angriffe auf die Transportinfrastruktur folgen den spektakulären terroristischen Überfällen in der ersten Hälfte der ersten Dekade nach 2000, besonders die berüchtigten Geiselnahmen im Dubrowka-Theater im Oktober 2002 sowie in einer Schule in Beslan im September 2004.

Zusätzlich zu diesen sporadischen Terrorattacken ist seit einigen Jahren ein wachsender Trend an Unsicherheit und sozialer Instabilität im Nordkaukasus zu beobachten. Die Region wird häufig als Herzland des innerstaatlichen Terrorismus in Russland bezeichnet. Terroranschläge sind hier die Regel und alltägliche Erscheinungen. In unterschiedlichem Ausmaß betrifft dies die Republiken Tschetschenien, Dagestan, Inguschetien und Kabardino-Balkarien. Soziale Unsicher- 
heiten und Spannungen breiten sich auch in anderen Teilen des Nordkaukasus aus, wie in Adygej, KaratschaiTscherkessien und sogar im Bezirk Stawropol, einem mehrheitlich von ethnischen Slawen bewohntem Gebiet (siehe den Beitrag von Andrew Foxall in den Russlandanalysen 218). Terroristische Aktivitäten und interethnische Spannungen nehmen in Russland weiter zu und betreffen einen immer größeren Teil des Landes und der Gesellschaft.

\section{Von einer existenziellen Bedrohung zum Sicherheitsrisiko}

Mit Beginn des zweiten Tschetschenienkriegs 1999 hat die Führung die Frage der inneren Instabilität Russlands als Kampf gegen den Terrorismus konstruiert. Terrorismus wurde als Erklärung für eine Reihe von Entwicklungen angeführt - vom zweiten Feldzug in Tschetschenien im Herbst 1999 bis hin zur Interpretation der Instabilität im Nordkaukasus. Die Versuche westlicher wie auch russischer Organisationen, darunter Memorial, diese Darstellungsweise durch den Verweis auf Themen jenseits des Terrorismus zu konterkarieren, zum Beispiel durch Hinweise auf gesellschaftliche Instabilität, die Lage der Menschenrechte, Polizeigewalt oder Probleme der Regierungsführung, sind jedoch gescheitert. Die russische Führung klammert sich an die Bezeichnung Terrorismus als eine Erklärung für alles.

\section{Terrorismus in den Zeiten Putins}

Das heißt jedoch nicht, dass sich Russlands offizielle Position im Krieg gegen den Terrorismus im letzten Jahrzehnt nicht verändert hätte. Der Standpunkt der Staatsführung und deren Konstruktion der terroristischen Bedrohung hat sich in der Tat bedeutend gewandelt. So auch die vorgeschlagenen Maßnahmen und Strategien zum Umgang mit dieser Bedrohung. In der Anfangsphase des Anti-Terrorkampfes von 1999-2001 betrieb die russische Führung eine securitization [d. h., eine "Versicherheitlichung", es wurde dem Problem eine besondere sicherheitspolitische Bedeutung zugewiesen; Anm. d. Red.] der Terrorgefahr, die von Tschetschenien ausging, und stellte sie als existenzielle Bedrohung sowohl des russischen Staates wie auch der größeren internationalen Gemeinschaft dar. Die Terrorgefahr wurde auf einheimische und internationale, islamistisch inspirierte Terrorgruppen zurückgeführt.

Die Lösung, die das Putin-Regime vertrat, war die Durchführung einer groß angelegten Militäroperation in Tschetschenien (Herbst 1999 bis Frühling 2000), das als Zentrum der terroristischen Bedrohung innerhalb Russlands galt. Es folgten kleinere Antiterror-Operationen von Anfang bis Mitte 2000er Jahre. Bei dieser Antiterror-Strategie ging es niemals darum, den Charakter der russischen Politik grundsätzlich zu ändern oder die staatliche Sicherheitspraxis in Reaktion auf eine zunehmende Unzufriedenheit in Tschetschenien zu korrigieren. Statt dessen waren die Operationen darauf ausgerichtet, das herrschende politische Regime und dessen Sicherheitsansatz in ganz Russland zu stärken, besonders in Gebieten, von denen man annahm, dass sie sich der russischen föderalstaatlichen Kontrolle entziehen wollten. Eine Änderung der innerhalb Russlands herrschenden Regeln wurde nicht erörtert, etwa durch Vorschläge, die Verhältnisse in Russland transparenter und fairer zu gestalten. Statt dessen wurde die Notwendigkeit eines starken Staates durch die Wiedereinführung von Kontrollen der Staatsinstitutionen betont.

Seit etwa 2005 wurde das tschetschenische Problem nicht länger als eine Gefahr für das Überleben Russlands angesehen - zumindest im Zusammenhang mit Terrorismus. Die russische Führung erklärte, die unmittelbare terroristische Bedrohung sei beseitigt, und es werde eine Politik der Normalisierung der politischen und sozioökonomischen Sphäre in Tschetschenien durchgesetzt, in Verbindung mit der Fortführung von Anti-TerrorOperationen (unter Führung von Achmad Kadyrow 2000-2004 und anschließend seines Sohnes, Ramsan Kadyrow, ab 2007).

Basierend auf dem öffentlich verkündeten Erfolg dieses Ansatzes in Tschetschenien, begann die russische Führung den Terrorismus als bloßes »Risiko« für die Sicherheit Russlands einzustufen und nicht mehr als grundsätzliche Bedrohung des russischen Staates. Daher wurden die fortgesetzten terroristischen Aktivitäten des sogenannten »Kaukasischen Emirats« unter Führung von Doku Umarow im ganzen Nordkaukasus und die Terroranschläge in russischen Städten den Überbleibseln der Terrorgruppen zugeschrieben, die auf Grund des Erfolgs der Normalisierungsstrategie aus Tschetschenien verdrängt worden seien. Diese ehemals in Tschetschenien aktiven Gruppen hätten sich, so hieß es, überwiegend nach Dagestan, Inguschetien und Kabardino-Balkarien zurückgezogen.

In Übereinstimmung mit dieser Neueinstufung der terroristischen Bedrohung wurde eine Reihe neuer Anti-Terror-Gesetze verabschiedet. Dazu zählt das neue Terrorismusbekämpfungsgesetz von 2006 und die Einrichtung des Nationalen Terrorbekämpfungs-Komitees (NAK) mit lokalen Zweigstellen in ganz Russland. Das NAK, so wurde behauptet, sei geeignet, mit dem herabgestuften "Terrorismusrisiko« mit Einsätzen in Form von kleineren Operationen umzugehen.

Während der zweiten Hälfte von Putins Präsidentschaft weigerte sich die russische Staatsführung, ihre Neuinterpretation des Terror-Gefahrenniveaus und ihren neuen Ansatz der Normalisierung aufzugeben. 
Weder die andauernde Kritik aus dem In- und Ausland an den Sicherheitseinsätzen im Nordkaukasus, an der Nichtbeachtung von Menschenrechtsfragen, am durchgehenden Versagen bei der Lösung der sozioökonomischen Probleme und der Fragen der politischen Steuerung, noch die Zunahme der Gewalt im gesamten Nordkaukasus, veranlassten das Putin-Regime, seine Vorgehensweise im Umgang mit der terroristischen Bedrohung zu ändern.

Das Desinteresse der russischen Bevölkerung bezüglich des Terrorismus - ganz im Gegensatz zum Optimismus angesichts des wirtschaftlichen Aufschwungs und des Zuflusses ausländischen Kapitals nach Russland um 2005 - bedeutete zudem, dass es kaum öffentlichen Druck auf die Regierung gab, ihre Herangehensweise an den Terrorismus zu ändern. Der Terrorismus wurde von 2005 bis 2010 im wesentlichen alleine deswegen als Risiko und nicht als direkte Bedrohung eingestuft, weil es nicht mehr das wichtigste Thema in Russland war.

\section{Medwedew - die Fortsetzung der bekannten Strategie}

Die Führung unter Präsident Medwedew (seit 2008) waren dagegen eher geneigt, das terroristische Problem ernsthafter einzustufen als zuvor. Dies hängt teilweise mit Medwedews Modernisierungsprojekt zusammen, aber auch damit, dass die eskalierende Gewalt im Nordkaukasus immer schwerer zu ignorieren war. In Reaktion auf die sich verschlechternde Lage im Nordkaukasus wurde eine Reihe unterschiedlicher Maßnahmen verabschiedet. Dazu zählen die Auswechselung von regionalen Administrationen (in Inguschetien), die Einrichtung des Föderalbezirks Nordkaukasus und die Ernennung von Alexander Chloponin zum Präsidialbevollmächtigten (er legte einen Plan zur wirtschaftlichen Entwicklung der Region innerhalb von 15 Jahren vor) sowie die fortdauernden Operationen zur Terrorismusbekämpfung in einigen Republiken der Region. Trotz dieser Korrekturen wird jedoch mehr oder weniger der gleiche Ansatz verfolgt, der die Notwendigkeit verbesserter wirtschaftlicher Bedingungen betont, die Probleme ordentlicher Regierungsführung verdrängt und sich auf Anti-Terror-Operationen vor Ort verlässt.

Obwohl unklar ist, inwieweit die von liberalen Kritikern der offiziellen Terrorbekämpfungsstrategie vorgeschlagen Maßnahmen Gewaltaktionen in Russland tatsächlich verringern würden, so ist zumindest klar, dass eine effiziente Umsetzung dieser liberalen Strategien eine radikale Erneuerung des russischen Staates sowie der Gesellschaft insgesamt notwendig machen würde. Trotz aller Modernisierungsrhetorik ist jedoch offensichtlich, dass die russischen Füh- rung zur Durchführung einer bedeutenden Erneuerung nicht bereit ist.

Die meisten russischen Terrorbekämpfungsstrategien des letzten Jahrzehnts, seien es groß angelegte Militäreinsätze um das Jahr 2000 oder eine Kombination von kleineren Anti-Terror Operationen und Normalisierungsstrategien um ein lokales autokratisches Regime, konzentrierten sich darauf, jede größere Veränderung der politischen Ordnung oder Gesellschaft Russlands $\mathrm{zu}$ verhindern, indem einzelne Korrekturen im Nordkaukasus durchgeführt wurden. Dieser Versuch, den status quo zu erhalten, hatte jedoch unbeabsichtigte Folgen, wie an der Zunahme des russischen Ultra-Nationalismus, wachsender Spannungen im gesamten Nordkaukasus sowie der Umgestaltung Tschetscheniens unter Präsident Kadyrow zu sehen ist.

Die russische Führung scheint zu akzeptieren, dass die gegenwärtige Strategie keine Verbesserung der Sicherheitslage erbracht hat, weder im Nordkaukasus noch in Russland insgesamt. Dennoch werden die gleichen Strategien beständig wiederverwendet. Das zeigt die Schuldzuweisung für die Ereignisse in Domodedowo an einzelne Beamte, anstelle einer Diskussion über die Schwäche der operativen Organe des russischen Staates insgesamt. Die russische Führung befindet sich, was das Konzept der Terrorismusbekämpfung betrifft, in einer Sackgasse. Eine radikal neue Lösung ist nicht in Sicht.

\section{Terrorismus als Alltag}

Ein Problem, das früher als außerordentliche Bedrohung dargestellt wurde, ist nun zur Regel (d. h. einer alltäglichen Erscheinung) in Russland geworden. Dies wird nicht nur von der russischen Führung so gesehen, sondern auch von der russischen Öffentlichkeit. Eine Meinungsumfrage des Lewada-Zentrums zeigt, dass sich die russische Bevölkerung damit abgefunden hat, mit dem Terrorismus zu leben. Der Studie zufolge äußerten seit 2005 50-60\% der Befragten die Ansicht, dass sich die Situation im Nordkaukasus nicht ändern werde. In einer weiteren Umfrage des Lewada-Zentrums von Januar 2011 stimmten ca. $48 \%$ der Befragten darin überein, dass Terroranschläge zu einem Bestandteil des Alltags in Russland geworden sind, $34 \%$ gaben an, dass die Häufigkeit von Terroranschlägen in Russland in Zukunft gleich bleiben werde. (vgl. Grafik 2 auf S. 12)

\section{Fazit}

Um das Problem des Terrorismus in Russland zu verstehen, muss man betrachten, wie sich die Frage des Terrorismus in der russischen Politik im letzten Jahrzehnt entwickelt hat. Die Interpretation der terroristischen Bedrohung durch die russische Führung hat in dieser Zeit drei Phasen durchlaufen: 1999- 
2004 securitization - Terrorismus als Bedrohung für die Fortexistenz des russischen Staates; 2004-2008 Terrorismus als Risiko, das ein wiedererstarkter Staat bewältigen kann; und ein konzeptionelles Durcheinander in der jüngsten Zeit ab 2008, da die Staatsführung kein eindeutiges Konzept zur Bewältigung des Problems mehr hat.

Allen Phasen ist gemeinsam, dass jeglicher Versuch, die Instabilität im Nordkaukasus oder die Gefahr vor Terroranschlägen in Russland zu überwinden, davon charakterisiert ist, dass eine umfassende Umstrukturierung der innenpolitischen Ordnung in Russland vermieden werden soll - eine Reform, die von vielen Experten empfohlen wird. Trotz Modernisierungversprechen durch Präsident Medwedew scheint eine Umkehr im Verhalten gegenüber Terrorismus und sozialer Instabilität unwahrscheinlich. Somit wird man weiter individuell auf terroristische Vorfälle reagieren, ohne eine umfassende und tiefgreifende Strategie zur Bekämpfung von Instabilität im Allgemeinen zu entwickeln.

Über die Autorin:

Aglaya Snetkov ist Senior Researcher am Center for Security Studies an der ETH Zürich. In Kürze erscheint ein Beitrag von ihr zu Russlands Sicherheitspolitik in der Zeitschrift Europe-Asia Studies. Sie arbeitet an einem Buch über die Entwicklung des russischen Sicherheitsdiskurses unter Putin und Medwedew und in einem Projekt zu post-sowjetischer regionaler Sicherheit und Afghanistan.

ANALYSE

\section{Asymmetrien der Terrorismusbekämpfung}

Michail Logvinov, Chemnitz

\section{Zusammenfassung}

Die russische Terrorismusbekämpfungspolitik hat nur unzureichende Erfolge. Dies liegt darin begründet, dass der russische Staat sich nicht ernsthaft mit dem extremistischen Umfeld und der Trägerideologie des Terrorismus auseinandersetzt. Darüber hinaus gibt es keine konsequente Strategie, um Terrorgruppen von ihren Finanzierungsquellen abzuschneiden. Bekämpfungsstrategien werden meist nicht zielorientiert eingesetzt. Mit Investitionen in Milliardenhöhe finanziert Moskau eine korrupte Elite im Nordkaukasus. Kurz, es fehlt nach wie vor eine zielgerichtete, konsequente Antiterrorpolitik, die die islamistische Ideologie wirkungsvoll delegitimiert, das extremistische und terroristische Milieu austrocknet sowie den Schutz der kritischen Infrastrukturen gewährleistet.

\section{Ungleichmäßigkeiten der russischen Terrorismusbekämpfung}

2003 erschien in der renommierten Moskauer rechtswissenschaftlichen Zeitschrift "Staat und Recht» ein Aufsatz des damaligen Generalstaatsanwalts Ustinow mit dem Titel »Die staatliche Antiterrorstrategie: allgemeine Konzeption und Rechtsaspekte«, der neuralgische Punkte der russischen Terrorismusbekämpfungspolitik thematisierte. Der Autor kritisierte, dass in Russland keine Lösungen für das Terrorismusproblem als kriminelles und sozialpolitisches Phänomen vorliegen. Diese Einschätzung ist in vielen Punkten nachzuvollziehen, denn die Konzepte zur Bekämpfung des Terrorismus weisen eine ganze Reihe von Schwächen auf.

\section{Terroristen ohne Eigenschaften?}

Eines der Probleme ist die Weigerung des russischen Staates, sich mit der Trägerideologie des Terrorismus auseinanderzusetzen. Trotz des nicht zu übersehenden islamistischen Referenzrahmens des nordkaukasischen Aufstandes bestehen muslimische Geistliche und vor allem das tschetschenische Republikoberhaupt Ramsan Kadyrow mit Nachdruck darauf, die Termini »Islamismus« oder »islamischer Extremismus« aus dem politischen Wortschatz zu streichen. So sprach Kadyrow sich auf einem Treffen der nordkaukasischen Muftis am 28. August 2009 dafür aus, in politischen $\ddot{\text { uußerungen und }}$ in den Medien den Ausdruck »islamischer Extremismus" zu vermeiden. Der Vorschlag wurde von Präsident Med- 
wedew unterstützt. Doch drei Tage später teilten Kadyrow vier festgenommene junge Dschihadisten mit, ihre Absicht sei es gewesen, als »Märtyrer« zu sterben.

Hätte Doku Umarow nicht seinen Anspruch auf ein Gebiet zwischen Schwarzem und Kaspischem Meer mit dem Namen »Emirat Kaukasus« kundgetan, hätte er nicht die "Feinde des Islam» (darunter die USA und Europa) als Feinde des »Emirats« identifiziert, hätte er sich nicht mit den "Mudschaheddin" außerhalb Russlands solidarisiert und hätten die Webauftritte des "Vilayets Dagestan« (»Dschamaat Shariat», seit dem 11. Mai 2011 »ilayat Dagestan«), des »Vilayets Inguschetien/Kaukasus-Emirat" (»Hunafa») und »des vereinigten Vilayets von Kabarda, Balkarien und Karatschai/ Kaukasus-Emirat« (»IslamDin«) sowie des KaukasusEmirates selbst (»Kavkazcenter«) nicht in regelmäßigen Abständen Aufrufe zum Dschihad und salafistische Propaganda mit dem symptomatischen salafistischen Vokabular veröffentlicht, hätte man noch ein Auge zudrücken und die islamistische Ideologie des Terrorismus und des Aufstandes im Nordkaukasus ausblenden können. Angesichts der eindeutigen Selbstbekenntnisse ist das jedoch ein Ding der Unmöglichkeit.

Im Kampf gegen die sogenannten "Banditen« weigern sich Moskau und die nordkaukasischen Terrorismusbekämpfer hartnäckig, den Gegner beim Namen zu nennen und seine Motivationslage zur Kenntnis zu nehmen. Im Gegenteil wird jene offenbar vorsätzlich missinterpretiert. Fehlerhafte Problemdiagnosen münden konsequenterweise in falsche Lösungsvorschläge und stehen somit der geistig-politischen Auseinandersetzung mit der islamistischen Ideologie im Weg. Die notorische Realitätsverweigerung und die Konstruktion von »Banditen bzw. Zombies ohne religiöse Inhalte im Kopf «, die allen Fakten widerspricht, verhindert zwangsläufig eine Strategie zur Delegitimierung des islamistischen Terrorismus in Russland.

Obwohl in Moskau und in Grosny nach wie vor von "Banditentum" gesprochen und jeder Bezug zum Islam ausgeblendet wird, leiten die Islamisten auf allen genannten Propaganda-Webseiten die Dschihadpflicht von religiösen Konzepten ab. Somit ignoriert die russische Terrorismusbekämpfungspolitik Gefahrenfaktoren wie Reichweite/Verankerung der Ziele in der Ideologie, Gewalttoleranz, Verbreitung in der Bezugsgruppe, ihre Attraktivität und Nachvollziehbarkeit. Dass Moskau keine Anstalten macht, die Trägerideologie des Terrorismus zu delegitimieren, liegt auch in der gültigen Definition des Terrorismus-Begriffs begründet. Denn das russische Terrorismusbekämpfungsgesetz (TBG) beschreibt Terrorismus nicht primär als Strategie (zweckgebundene Gewaltanwendung), sondern als "Gewaltideologie und -praxis«, was offensichtlicher Nonsens ist. Denn Terroris- mus an sich stellt noch keine Ideologie dar. Das ist lediglich ein modus operandi terroristischer Akteure, deren Motivationslage bzw. ideologisches Substrat die Gewaltanwendung erst ermöglicht. Die Bestimmung des Terrorismus als Ideologie blendet somit die entsprechende Trägerideologie des Terrorismus und mithin Legitimierungsquellen, Ziele und erhoffte Gratifikationen aus.

\section{Die Finanzierung des Terrorismus}

Ähnlich inkonsistent verhält es sich mit der Strategie zum Austrocknen der Finanzierungsquellen. Während Moskau und vor allem Grosny immer wieder erklärten, man ginge gegen die Finanzierung von außen vor, versagten die Sicherheitsbehörden beim Schutz der regionalen Unternehmer vor militanten Gewaltakteuren. Spätestens seit 2005 entwickelten sich die Spenden für den Dschihad (der sogenannte "Sakat») zu einer der lukrativen Finanzierungsquellen des islamistischen Terrorismus in Russland. Aufrufe oder Forderungen von "Emiren« werden zwecks Audiovisualität den Zielpersonen auf USB-Sticks zugestellt. Wer nicht zahlt, hat mit Folgen zu rechnen und muss um sein Leben fürchten. Die regionalen Sicherheitsbehörden versag $(t)$ en allerdings nicht nur hinsichtlich der Schutzpflicht. Auch bei der Strafverfolgung des Delikts "Terrorismusfinanzierung « im Fall einer freiwilligen Unterstützung haben der Inlandsgeheimdienst und das Innenministerium nachweislich nur geringe Anstrengungen unternommen. Nach Angaben der Staatsanwaltschaft gab es bis 2011 keine Strafsachen wegen Terrorismusfinanzierung. Vor diesem Hintergrund wirken Meldungen, dass auch regionale Politiker "Sakat" an die militanten Akteure abführ $(\mathrm{t})$ en, besonders alarmierend. Dergestalt hätte Moskau indirekt die Strukturen des islamistischen Terrorismus in Russland mitfinanziert.

Hin und wieder teilen der Inlandsgeheimdienst FSB und das Innenministerium mit, den Fahndern sei es gelungen, Zellen der illegalen Geldtransfersysteme (auch Hawala-Finanzsystem genannt) aufzudecken. Doch gemessen an der Arbeitsmigration aus Nordkaukasus oder Zentralasien und der Unterentwicklung des Bankwesens in den jeweiligen Republiken mitsamt den unzureichenden personellen Ressourcen, die den Sicherheitsbehörden zur Verfügung stehen, lässt sich vermuten, dass ein Gros der Schattengeldtransfers - und somit auch ein Teil der Terrorismusfinanzierung - nach wie vor über das Hawala-System fließt.

\section{Das Versagen der politischen Elite im Nordkaukasus}

Im Artikel "Milliarden verjuxt« zitiert am 7. Juli 2010 die Informationsagentur RIA Nowosti ein Statement vom Regierungschef Putin, demzufolge Moskau im ver- 
gangenen Jahrzehnt 800 Mrd. Rubel in den Nordkaukasus transferiert hat. Doch sei es nicht gelungen, die Situation der lokalen Wirtschaft grundlegend zu verändern. Hunderte von Milliarden an Transferleistungen zu »verjuxen" ist schon schlimm genug. Doch ein großer Anteil dieser Mittel ist auch in falsche "Kanäle» geflossen. Anstatt soziale und ökonomische Modernisierung zu fördern, hat der Kreml mit Investitionen in Milliardenhöhe regionale Regierungen und „Eliten« (lies Klans) aufgebaut und unterhalten, die eine Machtvertikale im Nordkaukasus gewährleisten sollten. Die regionalen Eliten lieferten zwar die erwünschten (manipulierten) Wahlergebnisse. Primär sind sie jedoch für die Intransparenz, Korruption und Vetternwirtschaft verantwortlich, die wiederum Wasser auf die Mühlen der islamistischen Propaganda sind. Denn schlechte Regierungsführung verschlimmert per definitionem die Lebensbedingungen der örtlichen Bevölkerung. 2010 formulierte die Regierung eine Strategie zur sozioökonomischen Entwicklung des nordkaukasischen Föderalbezirkes bis 2025, die weitere Milliardeninvestitionen unter besserer Kontrolle vorsieht. Diese werden jedoch von denselben korrupten Eliten verwaltet, die der Kreml seit Jahren der Misswirtschaft bezichtigt.

Zudem treiben die regionalen Eliten und Geistlichen die Theokratisierung und Islamisierung im Nordkaukasus voran. Doch der Kalkül, die Stärkung des "traditionellen" sufistisch-hanafitischen Islam würde den Islamisten den Wind aus den Segeln nehmen, geht nicht auf. Denn Salafisten (oder muwahhidun, Bekenner der Einheit Gottes, in Russland als "Wahhabiten« bekannt) akzeptieren den "traditionellen" Islam (welcher Rechtsschule oder Strömung auch immer) ebenfalls nicht. Anstatt in theologischen Gesprächen die Jugend von der religiös motivierten Gewalt abzubringen, kümmer(te)n sich die nordkaukasischen "Gebieter" mehr um politische Macht. Unbequeme Personen und Sinnsucher melde(te)n sie dem FSB als angebliche "Wahhabiten", gegen die der Staat nach wie vor mit aller Härte vorgeht. Es ist daher wenig verwunderlich, dass die jüngere Generation der russländischen Muslime angesichts dieses doppelten Versagens sowohl dem Staat als auch dem traditionellen Islam den Rücken kehrt und sich den islamistischen Organisationen und militanten Dschamaaten annähert.

\section{Die Sicherheitsapparate im Kampf gegen den Terrorismus}

Da die Delegitimierung der islamistischen Trägerideologie nicht gelingt, Maßnahmen zur Vorbeugung des Extremismus daher nicht erfolgreich sind, und da die Sicherheitsbehörden auch nicht immer imstande sind, das extremistische Umfeld mit gebotener Sorg- falt zu beobachten sowie die Radikalisierungsprozesse zu registrieren, stehen die Sicherheitsapparate vor der Aufgabe, den Kampf gegen bereits radikalisierte und/ oder militante Akteure zu führen. Einer der Gründe für die mangelhafte Präventionsarbeit ist im russischen TBG zu suchen, versteht doch die russische Kriminalpolitik die Terrorismusprävention als "Durchführung eines zielgerichteten Maßnahmenkomplexes zur Beseitigung der sozialen Gefahr eines einsetzenden Aktes des Terrorismus".

Der FSB und das Innenministerium führen beinahe täglich kontraterroristische Operationen in Wäldern und Dörfern wie Städten durch. Obwohl die russischen Terrorismusbekämpfer durchaus einige militärische Erfolge für sich verbuchen konnten, wachsen die abgeschlagenen Köpfe der nordkaukasischen TerrorHydra schnell nach. Die islamistischen Akteure erfreuen sich eines großen Zulaufs junger Dschihadisten - nicht zuletzt wegen der gegnerzentrierten Dominante der operativ-militärischen Terrorismusbekämpfung. Denn das primäre Ziel der russischen Sicherheitsbehörden ist es offensichtlich nicht, die Zivilbevölkerung zu schützen, sondern den Gegner - koste es was es wolle - zu vernichten. Das Ziel gilt auch dann als erreicht, wenn infolge der (unverhältnismäßigen) Gewaltanwendung weitere Personen sich radikalisieren. Während des mit massiver Gewalt ausgetragenen zweiten »Tschetschenienkrieges» verfolgte das Militär offensichtlich eine Abschreckungsstrategie, in der die Bestrafung (Tötung militärischer Führer und Feinde) und "Verhinderung (Zerstörung der terroristischen Infrastrukturen, die in einem asymmetrischen Konflikt nicht von zivilen Infrastrukturen zu unterscheiden sind) überproportional angelegt worden waren. Bis heute haben die Sicherheitsapparate nicht verinnerlicht, dass sie vor allen Dingen die Bevölkerung vor Terrorismus schützen müssen, anstatt sie in Mitleidenschaft zu ziehen. Einsätze der Sicherheitsapparate wirken sich sehr oft negativ auf die Lebensverhältnisse der Zivilbevölkerung aus. Die am 21. Februar 2011 in Kabardino-Balkarien angelaufene kontraterroristische Operation ist dafür ein aktuelles Beispiel. Denn die terroristischen Akteure strebten mit ihren Aktionen offenbar an, den Tourismus in der Region zu stören und Touristen zu verjagen, weshalb der Baksanski-Dschamaat neben einer Seilbahn auch eine Hotelanlage als Ziel ausgesucht und aus Moskau stammende Touristen direkt angegriffen hat. Die Sicherheitsbehörden spielen dabei mit ihren restriktiven Antiterrormaßnahmen den Terroristen in die Hände.

\section{Der Schutz kritischer Infrastrukturen}

Man muss kaum auf die Schwächen beim Schutz kritischer Infrastruktur hinweisen. Es hat wiederholt 
Anschläge auf Verkehrsinfrastruktur gegeben (etwa die wiederholten Anschläge auf den Zug Moskau-St. Petersburg und die Moskauer Metro sowie der Anschlag am Flughafen Domodedowo). Nach jedem Anschlag außerhalb des Nordkaukasus hat die Politik neue Programme vorbereitet und effizientere Maßnahmen versprochen. Doch Beobachter können sich des Eindrucks nicht erwehren, dass Moskau keinen systematischen Ansatz der Terrorismusbekämpfung hat und mit seinen ad hoc-Entscheidungen gewissermaßen von einem Terroranschlag zum nächsten stolpert.

Während die politischen Spitzenrepräsentanten die Verbesserung des physischen Schutzes kritischer Infrastruktur einfordern, überraschte der Inlandsgeheimdienst FSB Ende 2010 die Öffentlichkeit mit einer neuen Initiative. Danach soll der Art. 5 des Gesetzes »Über das Staatsgeheimnis", der auflistet, was als geheim gilt, um die Informationen über den Schutz der kritischen Infrastruktur erweitert werden. Im November 2010 hat das Parlament die Änderungen verabschiedet. Informationen über Maßnahmen zur Sicherung von potentiell gefährdeten Objekten, aber auch »Informationen über Kräfte, Mittel, Methoden, Pläne und Ergebnisse der Tätigkeit der zuständigen Behörden bei der Terrorbekämpfung sowie die Ergebnisse der finanziellen Überwachung von an Terroraktivitäten beteiligten Organisationen und Personen" gelten seitdem als Staatsgeheimnisse.

\section{Ziel-Mittel-Konflikte des Bekämpfungsansatzes}

Die Schwächen der russischen Terrorismusbekämpfungspolitik bestätigt auch ein Blick in das seit Ende 2009 gültige Konzept der Terrorismusbekämpfung in der Russländischen Föderation. Die Regierung hat es nach einer Dekade Antiterrorkrieg nicht vermocht, eine schlüssige, nicht formalistische und alle Gefahrenfaktoren umfassende Strategie zur Bekämpfung des transnationalen Terrorismus auszuarbeiten. Es mangelt in erster Linie an einer expliziten Abgrenzung der Gefährdung: Wer sind die Akteure und wie ist ihre Motivationslage? Welche begünstigenden Faktoren liegen vor, wie verwundbar sind die möglichen Anschlagsziele? Im Dokument fehlt überdies eine Risikobewertung, auf der die strategischen Maßnahmen aufbauen können. Auch ist im Konzept durchgehend vom »(modernen) Terrorismus" die Rede, der weder nach der Motivationslage der Akteure noch nach eingesetzten Mitteln und Metho- den näher definiert ist. Das Papier suggeriert den Eindruck eines auf Russland von außen einwirkenden Terrorismus, dessen Ausbreitung durch die Beseitigung der inneren »Ermöglichungsfaktoren" verhindert werden kann. Der ideologische Hintergrund des Terrorismus im Nordkaukasus wird in der Formel "gewisse historische Prämissen« versteckt. Es fehlen im Konzept auch Überlegungen, wie Zweck-Mittel-Konflikte des Bekämpfungsansatzes zu lösen sind. Exemplarisch sind an dieser Stelle die Beeinträchtigungen der strukturellen Terrorismusbekämpfung zu nennen, die durch einen unverhältnismäßigen Einsatz repressiver polizeilicher und militärischer Bekämpfungsmaßnahmen entstanden sind. Die Geheimhaltung von Mitteln, Methoden und Ergebnissen der Terrorismusbekämpfung deutet wiederum darauf hin, dass die Entscheidungsbefugnisse in Bezug auf die sachliche Abwägung zu schützender Rechtsgüter und Eingriffe in die Grundrechte nach wie vor den überforderten Sicherheitsdiensten überlassen werden. Dies ist eine der normativen Asymmetrien der Terrorismusbekämpfung, die oft dazu führt, dass die mit dem Islamismus und ausufernder Kriminalität konfrontierte Bürokratie sowie die durch die terroristische Gewalt herausgeforderten Sicherheitsbehörden am Rande der Selbst-Delegitimierung agieren.

\section{Fazit}

Bereits 2003 hatte der damalige Generalstaatsanwalt Ustinow die Schwächen der Terrorismusbekämpfung benannt. Er plädierte für eine multikausale und multidimensionale, über die repressiven Ansätze hinausgehende Bekämpfungsstrategie. Verblüffenderweise stimmen die Befunde aus aktuellen Untersuchungen mit seinen Diagnosen aus dem Jahr 2003 - von der Vorenthaltung strategischer Ressourcen über Auflösung terroristischer Strukturen bis hin zu Koordination und Kooperation der einzusetzenden Kapazitäten - größtenteils überein. Dies legt Zeugnis von einer niedrigen Intensität der staatlichen Entscheidungsfindung und Asymmetrien der Schwerpunktsetzung in diesem Politikfeld ab.

Als die Regierung 2009 ihr Terrorismusbekämpfungskonzept und 2010 eine Strategie zur sozioökonomischen Modernisierung der nordkaukasischen Region vorstellte, schien sich eine Kehrtwende abgezeichnet zu haben. Diese wird jedoch kraft genannter Asymmetrien keine deutliche Verbesserung bewirken können.

\section{Über den Autor}

Michail Logvinov studierte Russistik, Pädagogik und Germanistik sowie Neuere und neueste Geschichte und Politikwissenschaft in Wolgograd und Chemnitz. 2004 promovierte er im Fach Philologie zum Dr. phil. Derzeit strebt er eine Zweitpromotion an und lehrt Politikwissenschaft an der TU Chemnitz.

Lesetipps zum Artikel finden Sie auf der nächsten Seite. 
Lesetipps

- Uwe Halbach: Russlands inneres Ausland. Der Nordkaukasus als Notstandszone am Rande Europas. SWP-Studien 2010/S 27, Oktober 2010 http://www.swp-berlin.org/fileadmin/contents/products/studien/2010_S27_hlb_ks.pdf

- Uwe Halbach: Antiterrorismus und Jihad im postsowjetischen Raum. SWP-Studien 2007/S 22, August 2007 http:// www.swp-berlin.org/fileadmin/contents/products/studien/2007_S22_hlb_ks.pdf

- Maciej Falkowski: Chechnya: Between a Caucasian Jihad and 'hidden' separatism. OSW Policy Briefs, Issue 13, Warsaw, January 2007 http://www.osw.waw.pl/sites/default/files/PUNKT_WIDZENIA_13.pdf

- Maciej Falkowski, Mariusz Marszewski: The 'Tribal Areas' of the Caucasus. The North Caucasus - an enclave of 'alien civilisation' within the Russian Federation. OSW Studies, Issue 3 4, Warsaw, April 2010 http://www.osw.waw. pl/sites/default/files/PRACE_34.pdf

- Michail Logvinov: Der Terrorist ohne Eigenschaften? Zur Delegitimierung des Terrorismus und geistig-politischen Auseinandersetzung mit der islamistischen Ideologie in Russland, in: Eckhard Jesse, Gerd Strohmeier, Roland Sturm (Hrsg.): Europas Politik vor neuen Herausforderungen, Opladen 2011 (im Druck)

\section{CHRONIK}

\section{Bürgerkriegsaktionen und Terrorakte in Russland seit Dezember 2010}

\begin{tabular}{|l|l|}
\hline 07.12.2010 & $\begin{array}{l}\text { Bei einem Einsatz der Sicherheitskräfte werden im Rajon Sunschensk (Inguschetien) vier Untergrund- } \\
\text { kämpfer getötet. }\end{array}$ \\
\hline 12.12.2010 & $\begin{array}{l}\text { Bei einem Spezialeinsatz der Sicherheitskräfte im dagestanischen Dorf Buglen werden drei Unter- } \\
\text { grundkämpfer getötet. }\end{array}$ \\
\hline 23.12.2010 & $\begin{array}{l}\text { Im Rajon Tschegemsk (Kabardino-Balkarien) werden zwei Mitarbeiter der örtlichen Miliz von Unbe- } \\
\text { kannten erschossen. }\end{array}$ \\
\hline 26.12.2010 & $\begin{array}{l}\text { Bei einem Einsatz der Sicherheitskräfte in Machatschkala (Dagestan) werden acht Untergrundkämp- } \\
\text { fer getötet, darunter der Anführer, der Emir von Machatschkala, Magomed Scheichow. }\end{array}$ \\
\hline 04.01.2011 & $\begin{array}{l}\text { In Schamchal, einem Vorort von Machatschkala (Dagestan), werden zwei Untergrundkämpfer von } \\
\text { mit vier mutmaßlichen Untergrundkämpfern. Alle vier werden getötet. } \\
\text { Sicherheitskräften getötet. }\end{array}$ \\
\hline 05.01.2011 & $\begin{array}{l}\text { Bei einem Spezialeinsatz von Sicherheitskräften in Chasawjurt (Dagestan) werden vier Untergrund- } \\
\text { kämpfer getötet, darunter der sogenannte »Amir« von Chasawjurt, Ruslan Makawow. Drei Mitarbei- } \\
\text { ter der Sicherheitskräfte werden bei dem Einsatz verletzt. }\end{array}$ \\
\hline 09.01.2011 & $\begin{array}{l}\text { Beim Angriff auf einen Kontrollposten in Grosny (Tschetschenien) kommen ein Wehrdienstleistender, } \\
\text { ein Zivilist sowie einer der Angreifer ums Leben. Acht weitere Personen werden verletzt. }\end{array}$ \\
\hline 13.01.2011 & $\begin{array}{l}\text { In Machatschkala (Dagestan) werden bei einem Feuergefecht zwei Untergrundkämpfer getötet. Auf } \\
\text { Seiten der Sicherheitskräfte wird ein Milizionär getötet, ein weiterer verletzt. }\end{array}$ \\
\hline 14.01.2011 & $\begin{array}{l}\text { Bei einer Spezialoperation der Sicherheitskräfte in Machatschkala (Dagestan) werden drei Untergrund- } \\
\text { kämpfer getötet, darunter eine Frau. }\end{array}$ \\
\hline 14.01.2011 & $\begin{array}{l}\text { Bei der Explosion einer Autobombe in Chasawjurt (Dagestan) kommen vier Personen ums Leben, fünf } \\
\text { weitere werden zum Teil schwer verletzt. }\end{array}$ \\
\hline 24.01.2011 & $\begin{array}{l}\text { Bei einem Selbstmordanschlag im Ankunftsbereich des Moskauer Flughafens Domodedowo werden } \\
\text { 35 Personen getötet, über 160 weitere zum Teil schwer verletzt. }\end{array}$ \\
\hline 26.01.2011 & $\begin{array}{l}\text { Bei der Explosion einer Autobombe vor einem Café in Chasawjurt (Dagestan) werden vier Personen } \\
\text { getötet, sechs weitere verletzt. }\end{array}$ \\
\hline
\end{tabular}




\begin{tabular}{|c|c|}
\hline 27.01.2011 & $\begin{array}{l}\text { Adam Gusejnow, Untergrundkämpfer und Stellvertreter des Amirs von Dagestan, wird mit seiner } \\
\text { Frau bei einem Spezialeinsatz der Sicherheitskräfte in der Nähe von Chasawjurt (Dagestan) getötet. }\end{array}$ \\
\hline 02.02 .2011 & $\begin{array}{l}\text { In Kabardino-Balkarien werden bei zwei Überfällen fünf Inspektoren der Straßenverkehrspolizei } \\
\text { ermordet. Nach Angaben des Republikpräsidenten Arsen Konokow hat sich die Zahl der Übergriffe } \\
\text { auf Sicherheitskräfte im Jahr } 2010 \text { im Vorjahresvergleich vervierfacht. }\end{array}$ \\
\hline 04.02 .2011 & $\begin{array}{l}\text { Bei einem Überfall auf einen Gefangenentransport werden in der russischen Teilrepublik Karatschai- } \\
\text { Tscherkessien drei Polizisten ermordet. Ein Gefangener wird befreit. }\end{array}$ \\
\hline 07.02.2011 & $\begin{array}{l}\text { Doku Umarow, Anführer des Untergrundkampfes im Nordkaukasus und selbsternannter «Emir des } \\
\text { kaukasischen Emirats« bekennt sich als Organisator des Selbstmordanschlags auf dem Moskauer Flug- } \\
\text { hafen Domodedowo, bei dem am 24. Januar } 35 \text { Personen ums Leben kamen. }\end{array}$ \\
\hline 08.02.2011 & $\begin{array}{l}\text { Beim Versuch, eine Dokumentenkontrolle durchzuführen, werden in Naltschik (Inguschetien) zwei } \\
\text { Sicherheitskräfte getötet. }\end{array}$ \\
\hline 09.02.2011 & $\begin{array}{l}\text { Bei einem Überfall auf eine Kreisabteilung für innere Angelegenheiten in Chasawjurt (Dagestan) wer- } \\
\text { den zwei Milizionäre getötet. }\end{array}$ \\
\hline 14.02 .2011 & $\begin{array}{l}\text { Bei zwei Selbstmordanschlägen auf den örtlichen Polizeiposten sowie auf einen Kontrollposten in } \\
\text { Gubden (Dagestan) werden zwei Sicherheitskräfte getötet und } 27 \text { weitere zum Teil schwer verletzt. }\end{array}$ \\
\hline 18.02 .2011 & $\begin{array}{l}\text { Bei einem Überfall auf einen Kleinbus auf dem Weg in das Skigebiet Prielbruse (Kabardino-Balka- } \\
\text { rien) werden drei Touristen aus Moskau getötet, zwei weitere verletzt. }\end{array}$ \\
\hline 22.02 .2011 & $\begin{array}{l}\text { Bei Gefechten zwischen Sicherheitskräften und Untergrundkämpfern im Rajon Elbrus (Kabardino- } \\
\text { Balkarien) wird ein Wehrdienstleistender getötet und sechs weitere Sicherheitskräfte zum Teil schwer } \\
\text { verletzt. Mitteilungen über drei getötete Untergrundkämpfer werden nicht offiziell bestätigt. }\end{array}$ \\
\hline 25.02 .2011 & $\begin{array}{l}\text { In Naltschik (Kabardino-Balkarien) greifen Untergrundkämpfer das Gebäude des föderalen Sicher- } \\
\text { heitsdienstes, dessen Dienstsanatorium und zwei Kontrollposten an. Bis zu } 15 \text { Sicherheitskräfte wer- } \\
\text { den verletzt. }\end{array}$ \\
\hline 28.02.2011 & $\begin{array}{l}\text { Chasu Batalow, seit Dezember } 2009 \text { Anführer der Untergrundkämpfer im tschetschenischen Rajon } \\
\text { Atschchoj-Martanowk wird von Sicherheitskräften festgenommen. }\end{array}$ \\
\hline 02.03 .2011 & $\begin{array}{l}\text { Bei einem Spezialeinsatz der Sicherheitskräfte unter Leitung des tschetschenischen Präsidenten Ram- } \\
\text { san Kadyrow im Rajon Leninskij in Grosny (Tschetschenien) wird Arbi Sigauri, Anführer der Unter- } \\
\text { grundkämpfer in der Siedlung Slepzowskaja (Inguschetien) getötet. Zwei Sicherheitskräfte werden bei } \\
\text { dem Einsatz verletzt. }\end{array}$ \\
\hline 03.03.2011 & $\begin{array}{l}\text { Doku Umarow, Anführer des Untergrundkampfes im Nordkaukasus und selbsternannter »Emir des } \\
\text { kaukasischen Emirats«, ruft in einer Videobotschaft die russischen Muslime zum »heiligen Krieg» } \\
\text { gegen die »Ungläubigen« "an allen Fronten« auf. }\end{array}$ \\
\hline 09.03.2011 & $\begin{array}{l}\text { An einer Bushaltestelle am Mitschurin-Prospekt in Moskau, gegenüber der Akademie des Inlandsge- } \\
\text { heimdienstes FSB, explodiert ein Sprengsatz. Es kommt niemand zu Schaden. }\end{array}$ \\
\hline 10.03 .2011 & $\begin{array}{l}\text { Bei einem Feuergefecht im Rajon Tschegemsk (Kabardino-Balkarien) werden drei Untergrundkämp- } \\
\text { fer getötet und zwei Sicherheitskräfte verletzt. }\end{array}$ \\
\hline 11.03 .2011 & $\begin{array}{l}\text { Der UN Sicherheitsrat setzt Doku Umarow, den Führer des Untergrundkampfes im Nordkaukasus } \\
\text { und selbsternannten «Emir des kaukasischen Emirats", auf die Liste der gefährlichsten Terroristen. } \\
\text { UN-Mitgliedsstaaten ist demnach jegliche Unterstützung, einschließlich der Bereitstellung von Waf- } \\
\text { fen und Finanzmitteln, untersagt. }\end{array}$ \\
\hline 28.03 .2011 & $\begin{array}{l}\text { Bei Luftangriffen und einer Bodenoffensive der russischen Streitkräfte werden im inguschetischen } \\
\text { Rajon Sunschensk } 17 \text { Untergrundkämpfer getötet und zwei Verdächtige im Zusammenhang mit dem } \\
\text { Selbstmordattentat am Flughafen Domodedowo festgenommen. Auf Seiten der Sicherheitskräfte wer- } \\
\text { den bei dem Einsatz drei Personen getötet. }\end{array}$ \\
\hline
\end{tabular}




\begin{tabular}{|c|c|}
\hline 11.04 .2011 & $\begin{array}{l}\text { Bei einem Überfall im Rajon Suntinsk (Dagestan) an der Grenze zu Georgien, werden fünf Mitar- } \\
\text { beiter des russischen Innenministeriums getötet und sieben weitere Polizisten verletzt. Bei der darauf } \\
\text { folgenden Spezialoperation werden drei mutmaßliche Anführer der Suntinsker Untergrundkämpfer } \\
\text { getötet. Auf ihrer Rückfahrt werden die Spezialeinheiten erneut angegriffen, zwei weitere Polizisten } \\
\text { kommen ums Leben, drei werden verletzt. }\end{array}$ \\
\hline 17.04 .2011 & $\begin{array}{l}\text { Bei einer Fahrzeugkontrolle werden im Rajon Lewaschinsk (Dagestan) vier Untergrundkämpfer von } \\
\text { Sicherheitskräften getötet. Unter den Getöteten befindet sich voraussichtlich der Anführer der dage- } \\
\text { stanischen Untergrundkämpfer, Israpil Walidschanow. }\end{array}$ \\
\hline 18.04 .2011 & $\begin{array}{l}\text { Bei einem Spezialeinsatz der Sicherheitskräfte in einem Vorort von Machatschkala (Dagestan) werden } \\
\text { zwei mutmaßliche Untergrundkämpfer getötet. }\end{array}$ \\
\hline 21.04 .2011 & $\begin{array}{l}\text { Im tschetschenischen Rajon Schalinsk werden zwei Untergrundkämpfer von Sicherheitskräften getö- } \\
\text { tet. Einer der Kämpfer war nach Angaben der russischen Sicherheitskräfte Gesandter von Al-Qaida } \\
\text { und jordanischer Staatsbürger. }\end{array}$ \\
\hline 25.04 .2011 & $\begin{array}{l}\text { Im Rajon Sumadinsk (Dagestan) kommt es zu Zusammenstößen zwischen Untergrundkämpfern und } \\
\text { Sicherheitskräften. Zwei Kämpfer werden getötet, nach Angaben des nationalen Anti-Terror-Komitees } \\
\text { war einer der Getöteten Anführer des »Gebirgssektors« der Kämpfer. }\end{array}$ \\
\hline 26.04 .2011 & $\begin{array}{l}\text { In Wolgograd explodiert vor dem Gebäude der städtischen Verkehrspolizei eine Bombe. Es kommt zu } \\
\text { keinen Verletzten. Wenige Stunden später wird in der Akademie des Innenministeriums in Wolgo- } \\
\text { grad ein Sprengsatz entdeckt, der jedoch gezielt gesprengt wird. }\end{array}$ \\
\hline 27.04 .2011 & $\begin{array}{l}\text { Bei einer Fahrzeugkontrolle werden in Naltschik (Inguschetien) zwei mutmaßliche Untergrundkämp- } \\
\text { fer getötet. }\end{array}$ \\
\hline 27.04 .2011 & $\begin{array}{l}\text { Bei einem Überfall durch Untergrundkämpfer werden im Rajon Smadinsk (Dagestan) zwei Sicher- } \\
\text { heitskräfte getötet und drei weitere verletzt. Bei der darauf folgenden Spezialoperation werden drei } \\
\text { Untergrundkämpfer getötet. }\end{array}$ \\
\hline 28.04 .2011 & $\begin{array}{l}\text { Bei einem Spezialeinsatz der Sicherheitskräfte werden in Machatschkala zwei Untergrundkämpfer } \\
\text { getötet. }\end{array}$ \\
\hline 29.04 .2011 & $\begin{array}{l}\text { Bei einem Spezialeinsatz der Sicherheitskräfte werden in der Grenzregion zwischen dem Bezirk Staw- } \\
\text { ropol und der Republik Kabardino-Balkarien fünf mutmaßliche Untergrundkämpfer getötet. }\end{array}$ \\
\hline 02.05 .2011 & $\begin{array}{l}\text { Nach Angaben des Nationalen Anti-Terrorismus-Komitees (NAK) wurden in Russland seit Beginn } \\
\text { des Jahres } 136 \text { Untergrundkämpfer von Sicherheitskräften getötet und } 243 \text { Kämpfer und deren Unter- } \\
\text { stützer festgenommen. }\end{array}$ \\
\hline 03.05 .2011 & $\begin{array}{l}\text { Bei der Explosion eines Sprengsatzes vor einer Abteilung des Innenministeriums im Moskauer Stadt- } \\
\text { teil »Samoskwaretsche« wird ein Polizist verletzt. }\end{array}$ \\
\hline 03.05 .2011 & $\begin{array}{l}\text { Nach Angaben des tschetschenischen Republikchefs Ramsan Kadyrow wurden im Süden der Repu- } \\
\text { blik zwei Untergrundkämpfer von Sicherheitskräften getötet. }\end{array}$ \\
\hline 08.05 .2011 & $\begin{array}{l}\text { Bei Zusammenstößen zwischen Untergrundkämpfern und Sicherheitskräften im Rajon Kisljarsk } \\
\text { (Dagestan) werden drei Kämpfer und ein Wehrdienstleistender getötet, zwei weitere Sicherheitskräfte } \\
\text { werden verletzt. }\end{array}$ \\
\hline 10.05 .2011 & $\begin{array}{l}\text { Bei einem Spezialeinsatz der Sicherheitskräfte in Naltschik (Kabardino-Balkarien) werden zwei Unter- } \\
\text { grundkämpfer, eine Frau sowie ein weiterer, mutmaßlicher Kämpfer getötet. }\end{array}$ \\
\hline
\end{tabular}




\section{Terrorismus und Terrorismusbekämpfung in der russischen öffentlichen Meinung}

\section{Umfragen des Lewada-Zentrums}

Grafik 1: Welches Motiv bewegte die Organisatoren der Anschläge auf dem Flughafen Domodedowo 2011 bzw. in der Moskauer Metro 2010? (Mehrfachnennungen möglich)

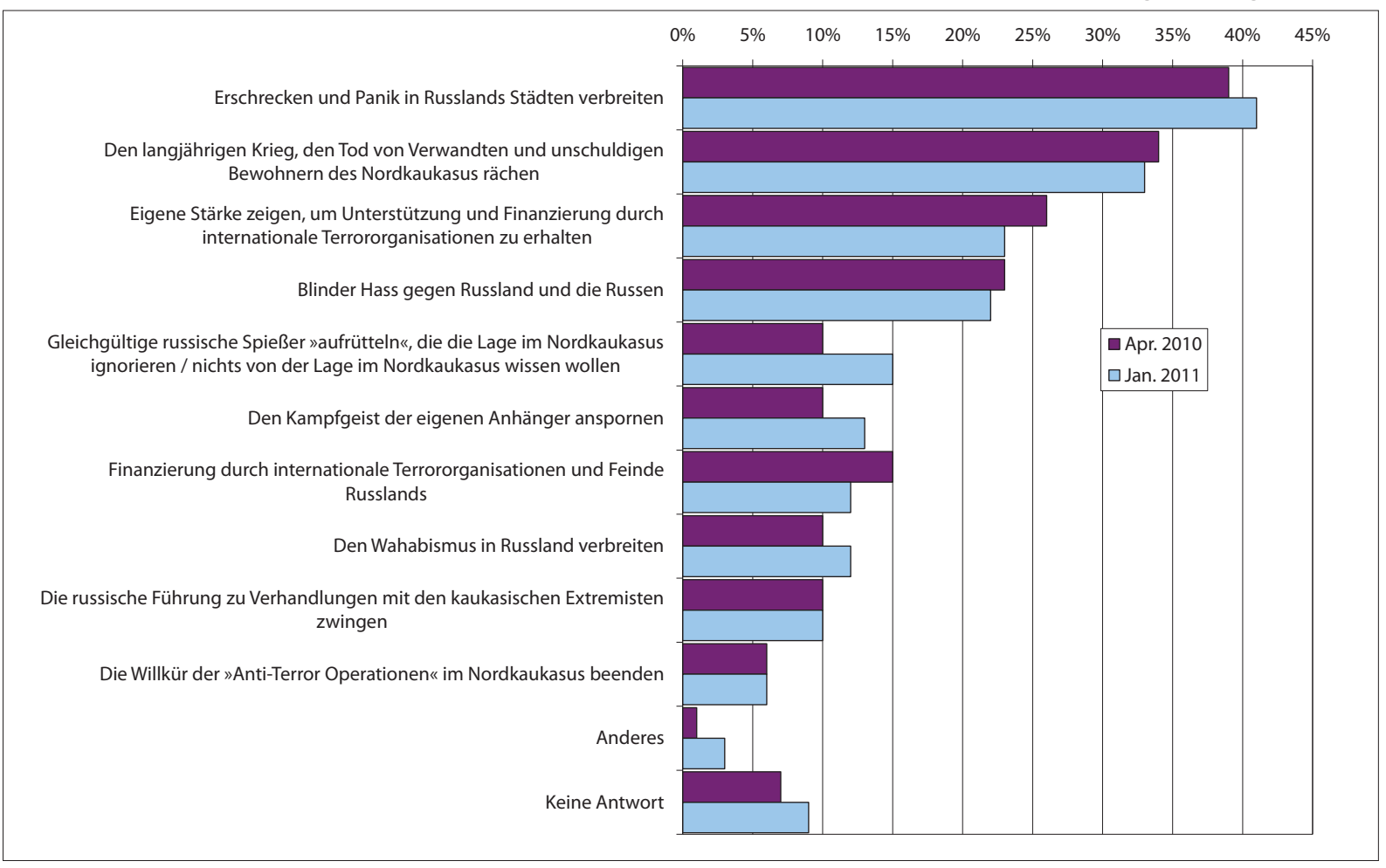

Quelle: Umfragen des Lewada-Zentrums vom 28.-31. Januar 2011 http://www.levada.ru/press/2011020404.html

Grafik 2: Stimmen Sie mit der Aussage überein, dass Terroranschläge zu einem gewohnten Teil unseres Lebens geworden sind?

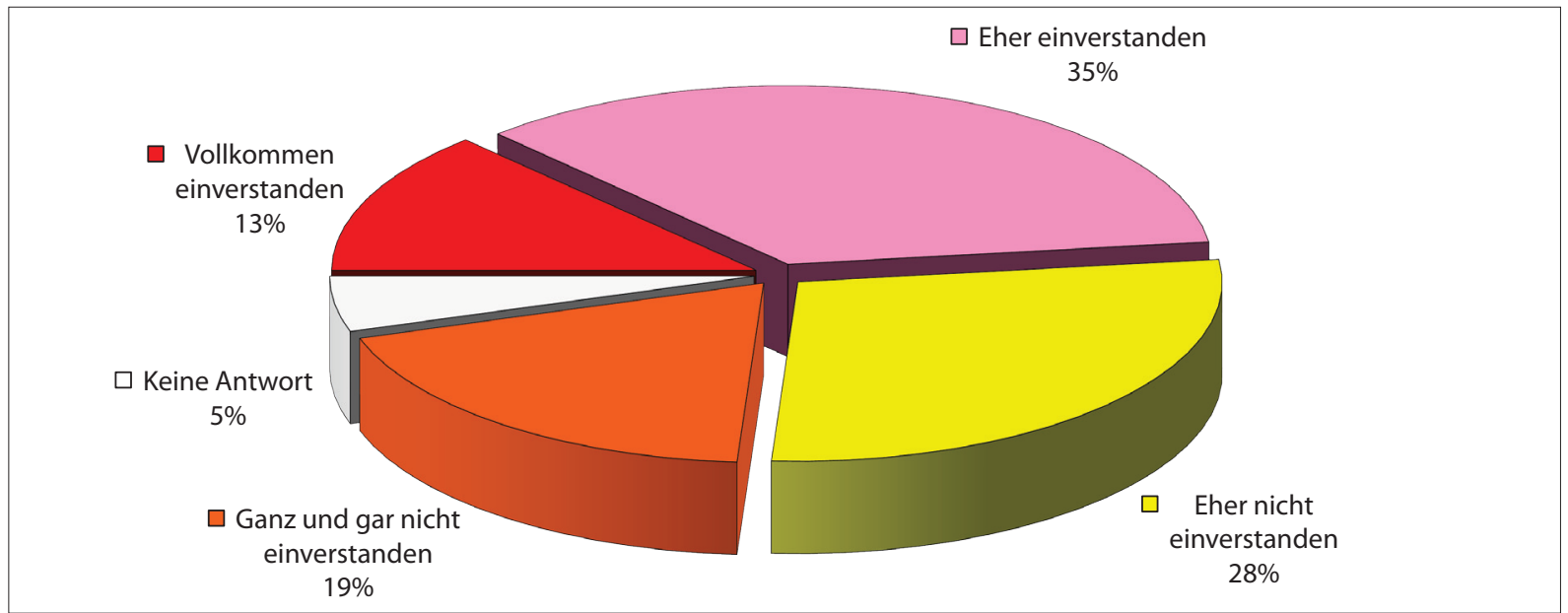

Quelle: Umfragen des Lewada-Zentrums vom 28.-31. Januar 2011 http://www.levada.ru/press/2011020404.html 
Grafik 3: Was sollte Ihrer Meinung nach zur Bekämpfung des Terrorismus unternommen werden? (Mehrfachnennungen möglich)

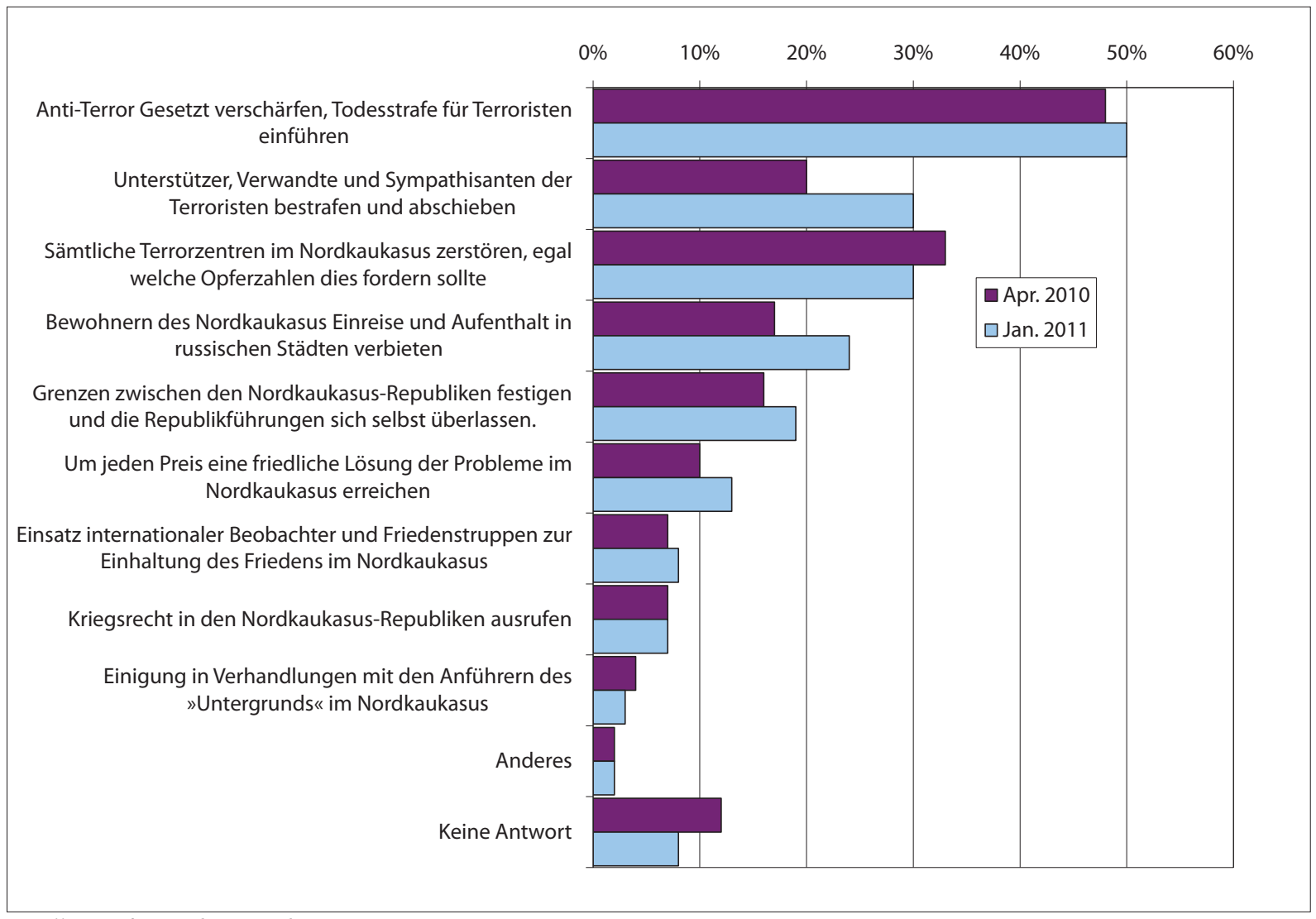

Quelle: Umfragen des Lewada-Zentrums vom 28.-31. Januar 2011 http:/www.levada.ru/press/2011020404.html

Grafik 4: Kann die russische Staatsmacht die Situation im Nordkaukasus unter Kontrolle bringen?

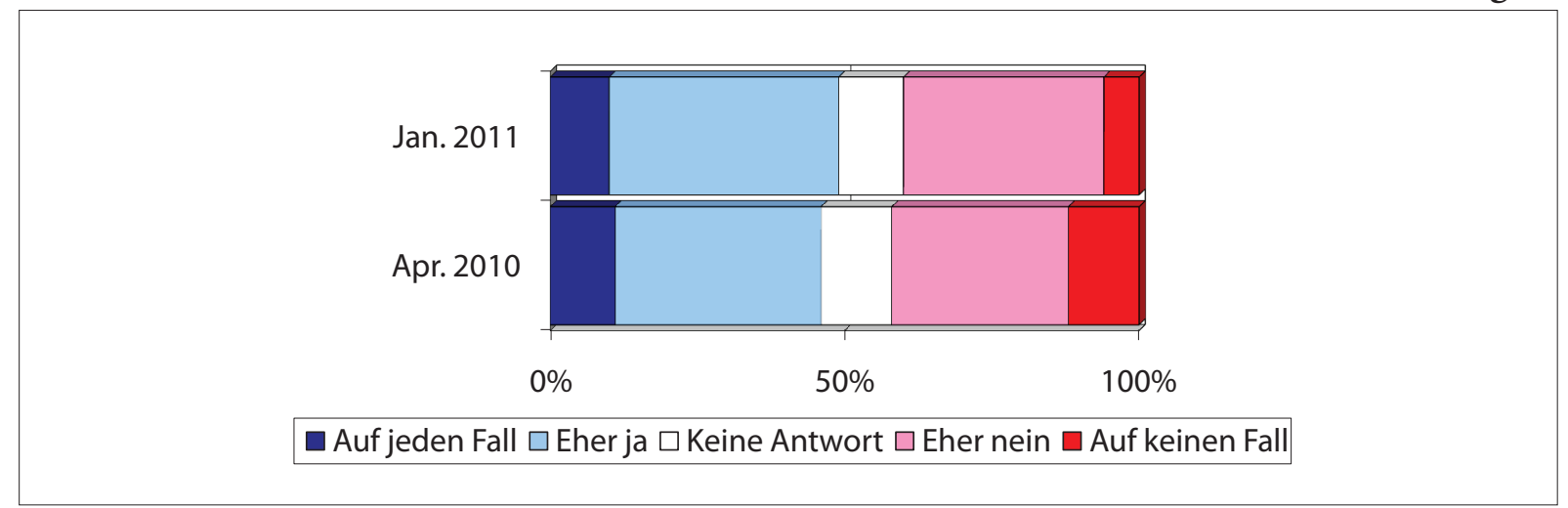

Quelle: Umfragen des Lewada-Zentrums vom 28.-31. Januar 2011 http://www.levada.ru/press/2011020404.html 
Grafik 5: Wie bewerten Sie die momentane Situation im Nordkaukasus?

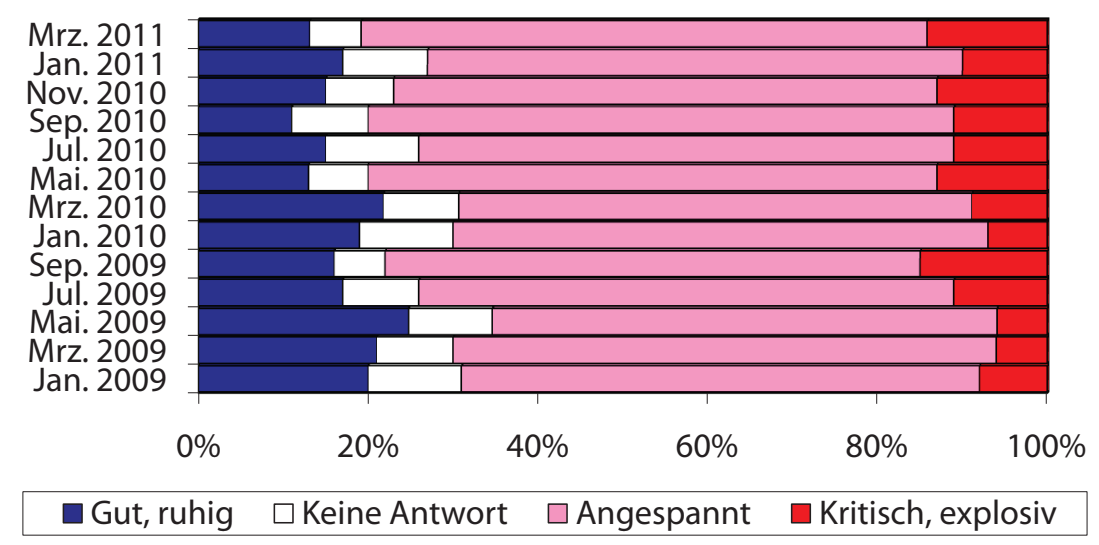

Quelle: Umfragen des Lewada-Zentrums vom 18.-21. März und vom 11.-14. Februar 2011 http://www.levada.ru/press/2011041802.html

Grafik 6: Wird sich die Situation im Nordkaukasus im kommenden Jahr ...

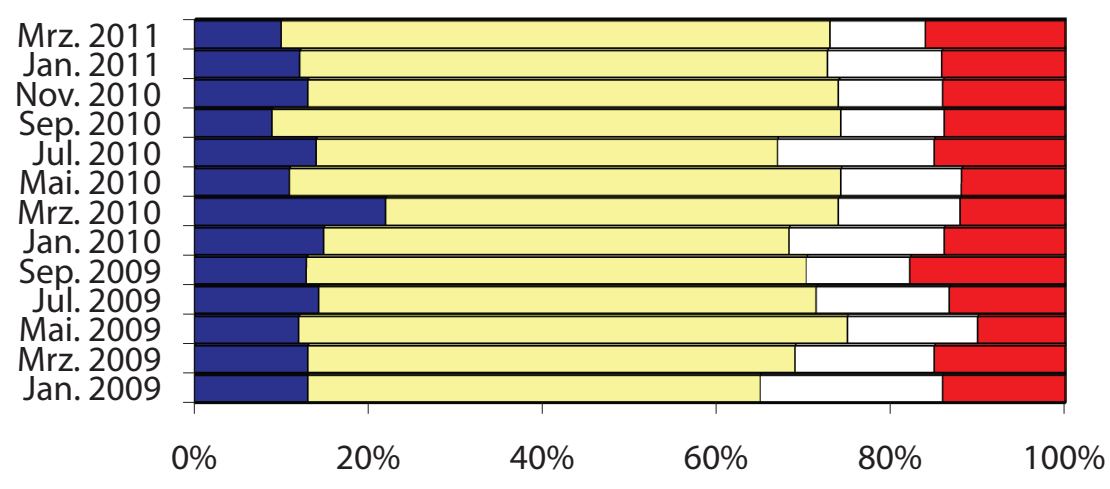

Verbessern $\square$ Gleich bleiben $\square$ Keine Antwort $\square$ Verschlechtern

Quelle: Umfragen des Lewada-Zentrums vom 18.-21. März und vom 11.-14. Februar 2011 http://www.levada.ru/press/2011041802.html

Grafik 7: Kontrolliert die Föderalmacht im Moment die Lage in Tschetschenien?

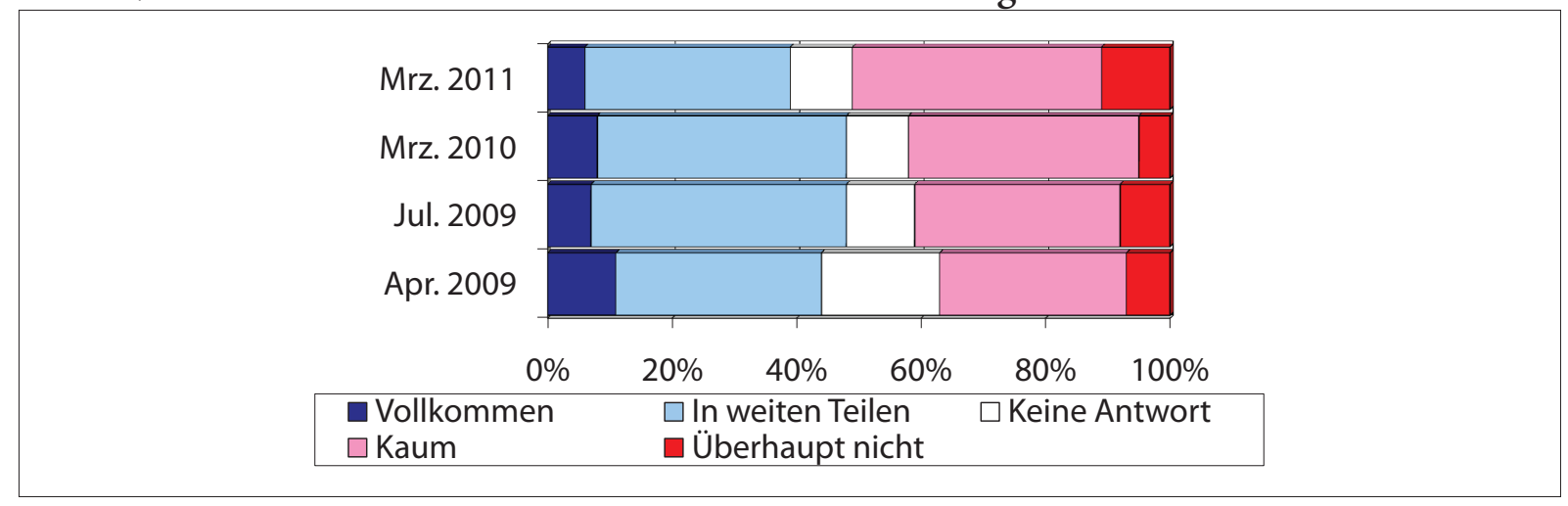

Quelle: Umfragen des Lewada-Zentrums vom 18.-21. März und vom 11.-14. Februar 2011 http://www.levada.ru/press/2011041802.html 


\section{Umfragen des WZIOM}

Grafik 8: Befürchten Sie zum gegenwärtigen Zeitpunkt, dass Sie selbst oder Ihnen Nahestehende Opfer eines Terroranschlages werden könnten?

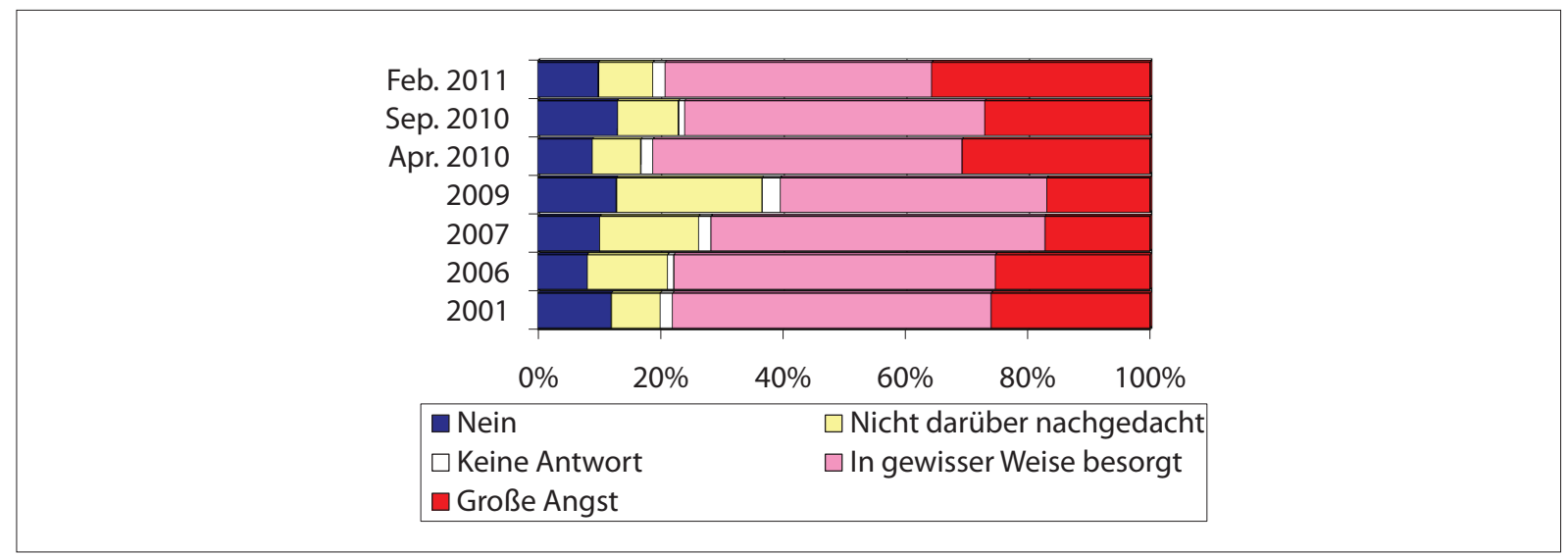

Quelle: Umfragen des WZIOM vom 5.-6. Februar 2011 http://old.wciom.ru/novosti/press-vypuski/press-vypusk/single/111350.html

\section{Umfragen des Lewada-Zentrums}

Grafik 9: Wird es in russischen Großstädten zu neuen Terroranschlägen und Geiselnahmen kommen?

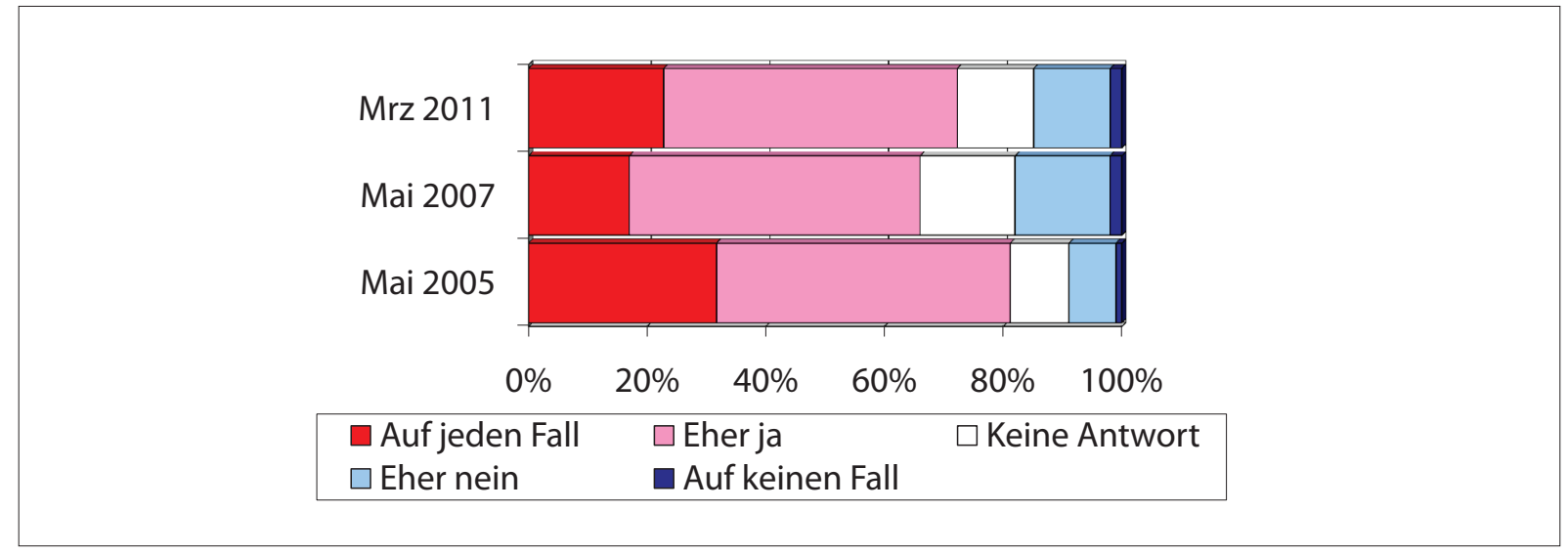

Quelle: Umfragen des Lewada-Zentrums vom 18.-21. März 2011 http://www.levada.ru/press/2011032901.html 
Grafik 10: Befürchten Sie, dass Sie selbst oder Ihnen Nahestehende, Opfer eines Terroranschlages werden könnten?

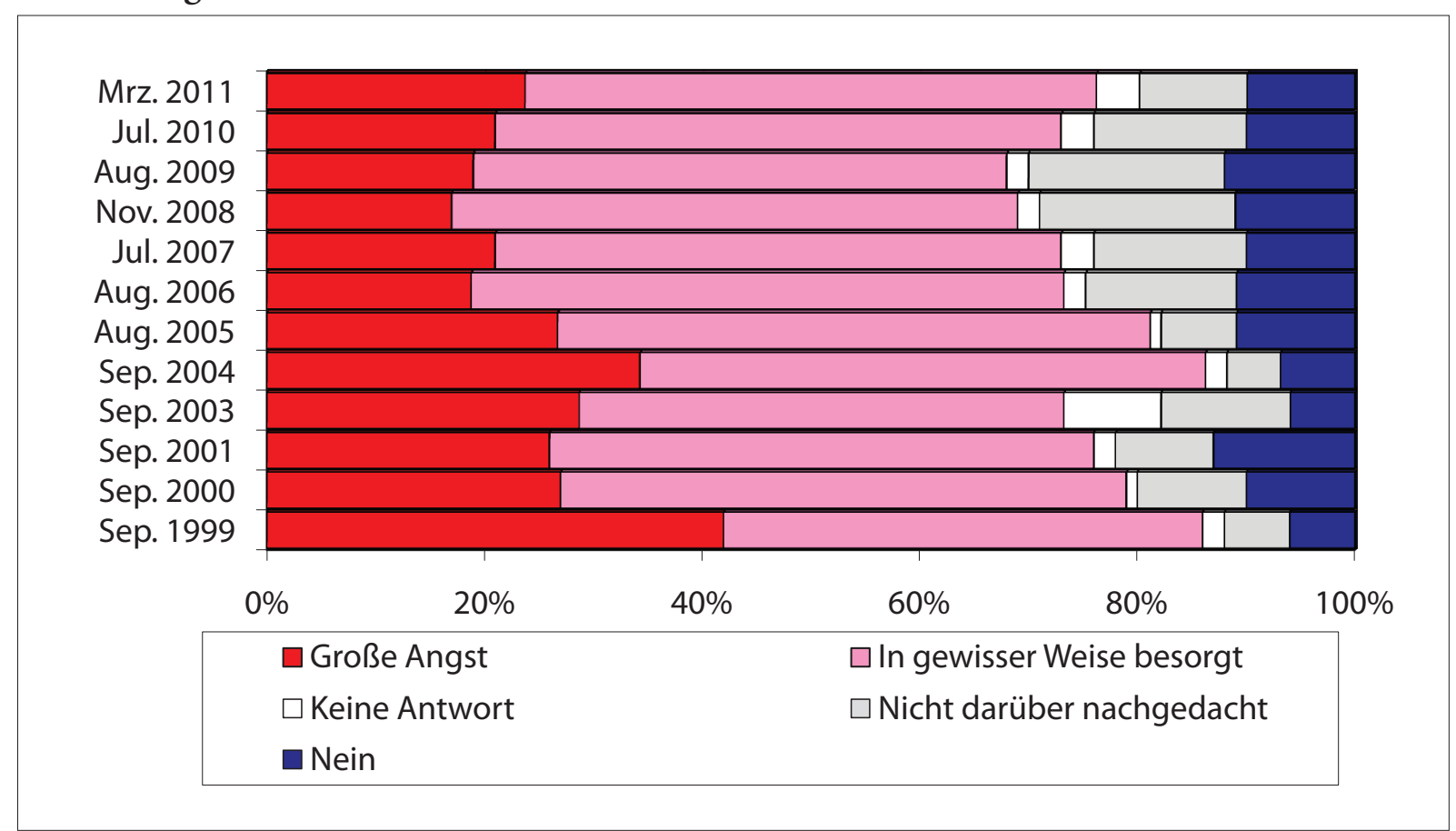

Quelle: Umfragen des Lewada-Zentrums vom 18.-21. März 2011 http://www.levada.ru/press/2011032901.html

Grafik 11: Können die russischen Spezialeinheiten und das Innenministerium die Bevölkerung vor neuen Terroranschlägen schützen?

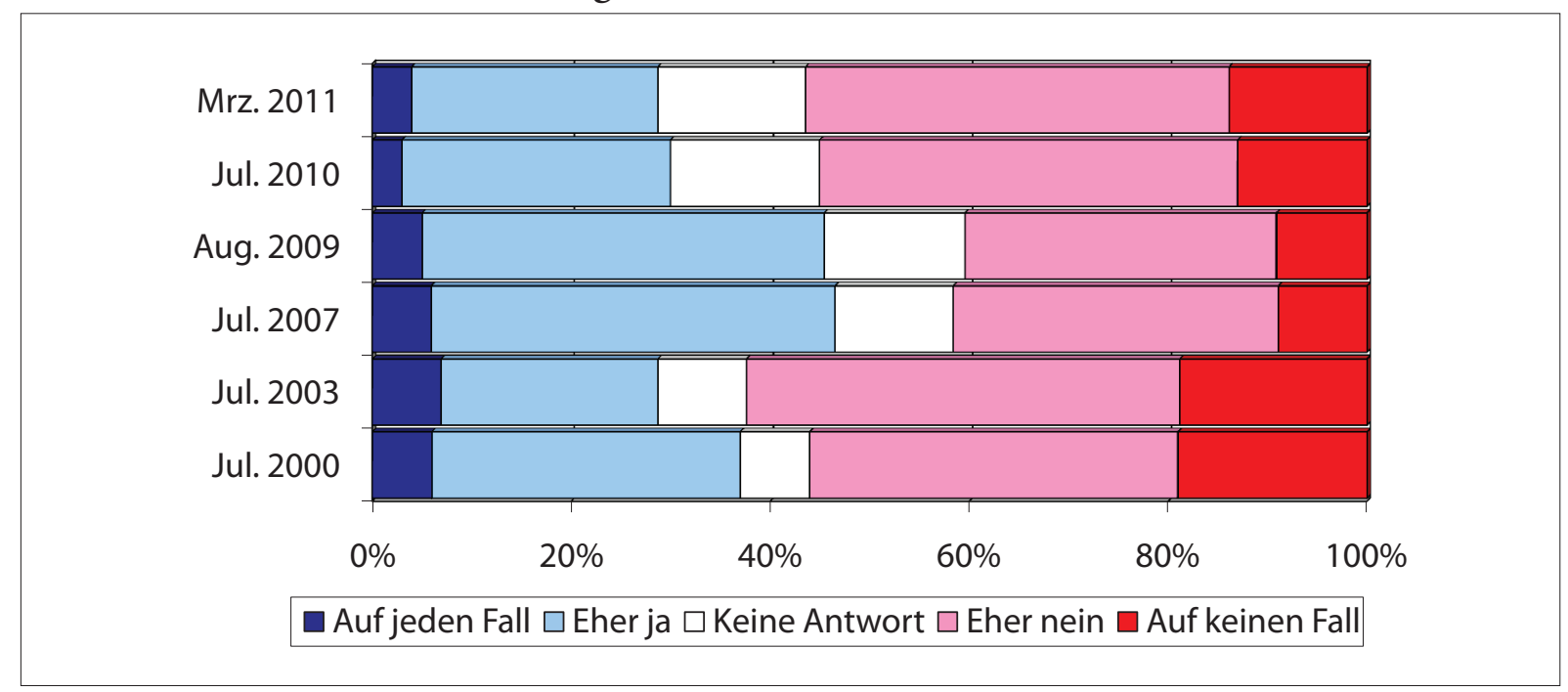

Quelle: Umfragen des Lewada-Zentrums vom 18.-21. März 2011 http://www.levada.ru/press/2011032901.html 


\section{Vom 5. bis zum 19. Mai 2011}

\begin{tabular}{|c|c|}
\hline 05.05 .2011 & $\begin{array}{l}\text { Mehrere russisch-nationalistische Bewegungen schließen sich zur gemeinsamen Organisation »Die Russen«zusam- } \\
\text { men. Der Vorsitz wechselt zwischen drei Personen, darunter ist der ehemalige Vorsitzende der im April verbo- } \\
\text { tenen »Bewegung gegen illegale Einwanderung«. Die Organisation ist bisher nicht offiziell registriert worden. }\end{array}$ \\
\hline 06.05 .2011 & $\begin{array}{l}\text { Das Moskauer Stadtgericht verurteilt Nikita Tichonow wegen des Mordes an der Journalistin Anastasija Barbu- } \\
\text { rowa und dem Anwalt Stanislav Markelow zu einer lebenslangen Haftstrafe. Seine Komplizin, Jewgenija Cha- } \\
\text { sis, wird zu } 18 \text { Jahren Haft verurteilt. Die beiden Angeklagten waren am 28.4. von den Geschworenen schul- } \\
\text { dig gesprochen worden. }\end{array}$ \\
\hline 06.05 .2011 & $\begin{array}{l}\text { Das Stockholmer Schiedsgericht gestattet einen Aktienaustausch zwischen BP und Rosneft unter der Bedin- } \\
\text { gung, das das Konsortium TNK-BP bei einem gemeinsamen Projekt zur Erschließung des arktischen Schelfs } \\
\text { beteiligt wird. }\end{array}$ \\
\hline 06.05 .2011 & $\begin{array}{l}\text { Ministerpräsident Wladimir Putin regt auf einer Parteikonferenz von »Einiges Russland« in Wolgograd an, eine } \\
\text { »Allrussische Volksfront« zu gründen. Neben »Einiges Russland» sollen dieser weitere Parteien, Gewerkschaf- } \\
\text { ten, Jugend- und Frauenorganisationen angehören. Im Rahmen der »Volksfront-Bewegung« sollen unabhän- } \\
\text { gige Kandidaten auf der Parteiliste von »Einiges Russland« bei der Dumawahl im Dezember antreten können. }\end{array}$ \\
\hline 07.05 .2011 & $\begin{array}{l}\text { Dmitrij Medwedew verleiht den Städten Starij Oskol, Kolpino und Anape die Ehrenbezeichnung "Stadt mili- } \\
\text { tärischen Ruhmes« für ihre mutige und standhafte Verteidigung im "Großen Vaterländischen Krieg». Diesen } \\
\text { Titel führen nun } 33 \text { russische Städte. } 12 \text { Städte werden als »Heldenstädte» bezeichnet, sieben davon befinden } \\
\text { sich auf russischem Staatsgebiet. }\end{array}$ \\
\hline 08.05 .2011 & $\begin{array}{l}\text { Bei Zusammenstößen zwischen Untergrundkämpfern und Sicherheitskräften im Rajon Kisljarsk (Dagestan) wer- } \\
\text { den drei Guerillas und ein Wehrpflichtiger getötet sowie zwei weitere Angehörige der Sicherheitskräfte verletzt. }\end{array}$ \\
\hline 08.05 .2011 & $\begin{array}{l}\text { Im Moskauer Stadtteil Chimki versammeln sich ca. } 200 \text { Menschen um gegen die Rodung des örtlichen Wal- } \\
\text { des für den Bau der Autotrasse Moskau - St. Petersburg zu demonstrieren. Einheiten der Spezialpolizei lösen die } \\
\text { Versammlung auf und nehmen } 30 \text { Personen vorübergehend fest, darunter Jewgenija Tschirikowa, Vorsitzende } \\
\text { der Bewegung zum Schutz des Waldes von Chimki, und Sergej Mitrochin, Vorsitzender der Partei "Jabloko«. }\end{array}$ \\
\hline 09.05 .2011 & $\begin{array}{l}\text { Am 66. Jahrestag des Siegs im "Großen Vaterländischen Krieg« findet in Moskau eine Siegesparade statt. } 20.000 \\
\text { Soldaten marschieren über den Roten Platz, gefolgt von Kampffahrzeugen und Waffensystemen, darunter die } \\
\text { Interkontinentalrakete Topol-M, T-90 Panzer und Militärhubschrauber. }\end{array}$ \\
\hline 10.05 .2011 & $\begin{array}{l}\text { Bei einem Spezialeinsatz der Sicherheitskräfte in Naltschik (Kabardino-Balkarien) werden zwei Untergrund- } \\
\text { kämpfer, eine Frau sowie ein weiterer, mutmaßlicher Kämpfer getötet. }\end{array}$ \\
\hline 10.05 .2011 & $\begin{array}{l}\text { Das Untersuchungskomitee der russischen Staatsanwaltschaft teilt mit, dass es ein Verfahren gegen den Blogger } \\
\text { Alexej Nawalnij eingeleitet hat. Ihm wird, in Zusammenhang mit dem staatlichen Holzwirtschaftsunterneh- } \\
\text { men Kirowles in der Region Kirow, die "Verursachung von Vermögensschäden durch Täuschung oder Vertrau- } \\
\text { ensmissbrauch« vorgeworfen. Nawalnij wurde durch die von ihm betriebene Internetplattform http://rospil.info } \\
\text { bekannt, die auf zweifelhafte Staatsaufträge hinweist. }\end{array}$ \\
\hline 11.05 .2011 & $\begin{array}{l}\text { In Grosny wird das neue Fußballstadion »Achmat-Chadschi Kadyrow«, benannt nach dem getöteten Vater des } \\
\text { aktuellen Republikpräsidenten Ramsan Kadyrow, feierlich eröffnet. Bei einem Freundschaftsspiel tritt eine All- } \\
\text { Star-Mannschaft an Weltfußballern, darunter Robbie Fowler, Diego Maradona, Steve McManaman, Franco } \\
\text { Baresi, Fabien Barthez und Luis Figo gegen eine Auswahl russischer und tschetschenischer Fußballer mit Ram- } \\
\text { san Kadyrow als Kapitän an. Kadyrows Team gewinnt die 40-minütige Partie mit 5:2. }\end{array}$ \\
\hline 12.05 .2011 & $\begin{array}{l}\text { Dmitrij Medwedew verlängert die Frist zur Neuattestierung der Mitarbeiter des Innenministeriums um zwei } \\
\text { Monate bis zum 1. August 2011. Am Vortag entließ Medwedew per Dekret den Leiter der Hauptverwaltung des } \\
\text { Innenministeriums im Fernöstlichen Föderalbezirk sowie den Leiter der Transportabteilung des Innenministe- } \\
\text { riums im Föderalbezirk Ural. }\end{array}$ \\
\hline 13.05 .2011 & $\begin{array}{l}\text { Die russische Staatsduma veröffentlicht die Einkommens- und Vermögenserklärungen ihrer Deputierten für das } \\
\text { Jahr 2010. Den Angaben zufolge ist Leonid Simanowskij, Erster Stellvertretender Vorsitzender des Dumako- } \\
\text { mitees für Energie, reichster Abgeordneter mit einem Einkommen im Jahr } 2009 \text { von umgerechnet ca. } 30 \text { Mio. } \\
\text { Euro. Ihm folgt Wladimir Grusdew, Erster Stellvertretender Vorsitzender des Dumakomitees für Zivil-, Straf-, } \\
\text { Schieds- und Prozessgesetzgebung, mit ca. } 24 \text { Mio. Euro und Grigorij Anikejew, Mitglied im Komitee für Arbeit } \\
\text { und Soziales, mit ca. } 22 \text { Mio. Euro. }\end{array}$ \\
\hline 13.05 .2011 & $\begin{array}{l}\text { Amnesty International veröffentlicht seinen Jahresbericht 2011. In Russland begegnen Menschenrechtler und } \\
\text { unabhängige Journalisten weiterhin Bedrohungen, Schikanen und Übergriffe; Ermittlungen hierzu führen meist } \\
\text { zu wenigen Ergebnissen. Die Versammlungs- und Redefreiheit wird häufig eingeschränkt, und die Sicherheits- } \\
\text { lage im Nordkaukasus bleibt angespannt. }\end{array}$ \\
\hline
\end{tabular}




\begin{tabular}{|c|c|}
\hline 13.05 .2011 & $\begin{array}{l}\text { ch Änderungen im Ordnungsrecht durch das Moskauer Stadtparlament erhöht sich die Strafe für Schwarz- } \\
\text { hrer in den öffentlichen Verkehrsmitteln Moskaus von } 100 \text { auf } 1000 \text { Rubel (2,5 Euro auf } 25 \text { Euro). }\end{array}$ \\
\hline 13. & $\begin{array}{l}\text { Jelena Lewina, Anwältin des ehemaligen Jukos-Vorsitzenden Michail Chodorkowskij, wird in Moskau überfal- } \\
\text { len. Ihr werden wichtige Dokumente für das bevorstehende Kassationsverfahren von Michail Chodorkowskij } \\
\text { und Platon Lebedew gegen das zweite Urteil vom 30. Dezember } 2010 \text { entwendet. }\end{array}$ \\
\hline 15.05 .2011 & $\begin{array}{l}\text { einem Fußballspiel zwischen »Krylja Sowjetow« und "Spartak Moskwa« in Samara kommt es zu Ausschrei- } \\
\text { ngen. Mehr als } 20 \text { Fußballfans werden verletzt und mehr als } 120 \text { Anhänger von Spartak vorübergehend } \\
\text { tgenommen. }\end{array}$ \\
\hline 16.05 & $\begin{array}{l}\text { Aktivisten der »ungen Garde«, der Jugendbewegung von »Einiges Russland«, demonstrieren vor der Moskauer } \\
\text { Zentrale der Kommunistischen Partei Russlands für eine Beerdigung Lenins und der Entfernung des Mauso- } \\
\text { leums vom Roten Platz. }\end{array}$ \\
\hline 16.05 & $\begin{array}{l}\text { einer offiziellen Erklärung erinnert der Sprecher des russischen Außenministeriums Belarus an seine Verpflich- } \\
\text { g zur Einhaltung der Menschenrechte. Eine Reihe von harten Urteilen gegen Teilnehmer der Demonstratio- } \\
\text { t vom 19. Dezember 2010, darunter die Verurteilung des damaligen Präsidentschaftskandidaten Andrej San- } \\
\text { cow, werfe in Moskau Fragen auf. }\end{array}$ \\
\hline 16.0 & r Unternehmer Russlands, erklärt seine Kandidatur für den Vorsitz der Par- \\
\hline 17.05 & richt verschiebt das Revisionsverfahren von Michail Chodorkowskij und Platon Lebe- \\
\hline 17.05 & $\begin{array}{l}\text { s Moskauer Bürgermeisteramt verbietet zum wiederholten Male die Durchführung einer Gay Pride-Parade } \\
\text { der russischen Hauptstadt. Zur Begründung werden unzählige Protestschreiben von gesellschaftlichen Verei- } \\
\text { ungen und »einfachen Moskauern« genannt. }\end{array}$ \\
\hline 17.05 & $\begin{array}{l}\text { e geplante Zusammenarbeit zwischen BP und Rosneft ist geplatzt. Rosneft zieht sich aus c } \\
\text { rück, nachdem eine Einigung mit dem Investitionskonsortium AAR von TNK-BP nicht erz }\end{array}$ \\
\hline 18.05 & $\begin{array}{l}\text { Auf Antrag der Partei »Einiges Russland« entzieht das Regionalparlament von St. Petersburg Sergej Mironow, } \\
\text { Mitglied der Partei »Gerechtes Russland« und deren ehemaliger Vorsitzender, das Mandat im Föderationsrat. } \\
\text { Mironow verliert dadurch sein Amt als Präsident des Föderationsrats, dem russischen Oberhaus. }\end{array}$ \\
\hline 18.05 & $\begin{array}{l}\text { Dmitrij Medwedew gibt eine große Pressekonferenz in der Wirtschaftshochschule in Skolkowo. Vor ca. } 800 \text { in- } \\
\text { und ausländischen Medienvertretern vermeidet er eine klare Aussage über eine Kandidatur für eine zweite Amts- } \\
\text { zeit, die Entscheidung werde jedoch bald fallen. Eine gemeinsame Kandidatur mit Wladimir Putin erklärt er für } \\
\text { unwahrscheinlich. Seiner Meinung nach haben er und Putin ähnliche Ansichten zur Modernisierung Russlands, } \\
\text { jedoch unterschiedliche taktische Herangehensweisen. Auf Chodorkowskij angesprochen erwidert Medwedew, } \\
\text { dass eine Freilassung des ehemaligen Jukos-Chefs keine Gefahr für Russland sei. }\end{array}$ \\
\hline 19.05 .2011 & $\begin{array}{l}\text { Ministerpräsident Wladimir Putin nimmt in Minsk am Treffen des Zwischenstaatlichen Rates der Zollunion } \\
\text { zwischen Russland, Kasachstan und Belarus und an Treffen der Regierungschefs der Eurasischen Wirtschaftsge- } \\
\text { meinschaft sowie der GUS teil. Bezüglich der Zollunion geht es um die Ausgestaltung des Gemeinsamen Wirt- } \\
\text { schaftsraumes ab 1. Januar 2012. Eine Einigung über den Vertrag der Freihandelszone wird auf Grund von Mei- } \\
\text { nungsverschiedenheiten auf ein nächstes Treffen der Regierungschefs verschoben. }\end{array}$ \\
\hline
\end{tabular}

Die Russland-Analysen werden vom Ost-Ausschuss der Deutschen Wirtschaft unterstützt.

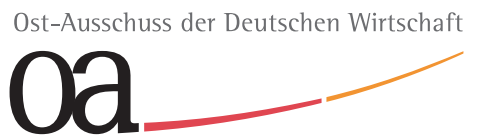

Herausgeber: Forschungsstelle Osteuropa an der Universität Bremen und Deutsche Gesellschaft für Osteuropakunde

Die Meinungen, die in den Russland-Analysen geäußert werden, geben ausschließlich die Auffassung der Autoren wieder. Abdruck und sonstige publizistische Nutzung sind nach Rücksprache mit der Redaktion gestattet.

Redaktion und Satz: Matthias Neumann, Heiko Pleines, Hans-Henning Schröder

Russland-Analysen-Layout: Cengiz Kibaroglu, Matthias Neumann und Michael Clemens

Die Russland-Analysen werden im Rahmen der Datenbank World Affairs Online (WAO) ausgewertet und sind im Portal IREON www.ireon-portal.de recherchierbar. ISSN 1613-3390 @ 2011 by Forschungsstelle Osteuropa, Bremen

Forschungsstelle Osteuropa • Publikationsreferat • Klagenfurter Str. 3 • 28359 Bremen • Telefon: +49 421-218-69600 • Telefax: +49 421-218-69607 e-mail: publikationsreferat@osteuropa.uni-bremen.de•Internet-Adresse: http://www.laender-analysen.de/russland/ 


\section{Abstracts aus Osteuropa 2-3/2011 »Logbuch Arktis«}

Die Arktis ist in den Fokus der internationalen Öffentlichkeit gerückt. Grund sind der rapide Klimawandel, die Ressourcen und die Hoffnung auf neue Verkehrswege. Gleichzeitig wächst die Sorge, dass aus den konkurrierenden Interessen Konflikte eskalieren könnten. Russland ist einer der zentralen Akteure im Raum. Osteuropa analysiert alle Aspekte im Heft 2-3/2011 »Logbuch Arktis. Der Raum, die Interessen und das Recht«. Der Band enthält 27 Beiträge auf 448 Seiten, 80 Abbildungen sowie 24 Karten und kostet 32,00 € (für Studenten 26,00 €). ISBN: 978-3-83051845-7 Bestellungen bitte über osteuropa@dgo-online.org

\section{Philipp Felsch}

Der arktische Konjunktiv

Auf der Suche nach dem eisfreien Polarmeer

In Frankenstein, einem Klassiker der phantastischen Literatur, findet sich die Verheißung von einem eisfreien Polarmeer, das ungeahnte Reichtümer birgt. Mitte des 19. Jahrhunderts übertrug der deutsche Geograph August Petermann den Mythos vom offenen Meer in eine exakte Theorie. Petermanns imaginäres Potential polarisierte. In der Populärliteratur fand er enormen Zuspruch, Fachleute reagierten skeptisch. Je stärker die Skepsis in offenen Anfeindung umschlug, desto fanatischer verfolgte Petermann seine Idee. Im Milieu der deutschen Nationalbewegung fiel sie auf fruchtbaren Boden. Es gelang ihm sogar, die deutsche Polarforschung ins Leben zu rufen. Petermanns Theorie erwies sich als falsch. Dass die Natur eines Tages seiner Theorie entgegenschmelzen könnte, hatte er in seinen kühnsten Träumen nicht zu hoffen gewagt.

\section{Jörg Stadelbauer}

\section{Naturraum Arktis}

\section{Russlands Hoher Norden}

Das internationale Interesse an der Arktis wächst. Hintergrund sind die regionalen Folgen des Klimawandels. In Teilen der Arktis ist die global bedeutendste Erwärmung des letzten halben Jahrhunderts zu beobachten. Das Abschmelzen des Meereises könnte die Erschließung von Bodenschätzen erleichtern und neue Schifffahrtsrouten eröffnen. Doch die Arktis ist mehr als ein Wirtschaftsraum. Sie ist ein höchst fragiles Ökosystem und Lebensraum zahlreicher indigener Völker. Arktis und Subarktis machen ein Fünftel der Festlandfläche Russlands aus. Russland ist einer der wichtigsten Staaten der Region und hat erheblichen Einfluss darauf, ob die ökologischen und ökonomischen Interessen in der Arktis kooperativ oder konfrontativ geregelt werden.

\section{Rüdiger Gerdes}

Klimawandel in der Arktis

Phänomene und Prognosen

Die eisbedeckte Fläche im Nordpolarmeer geht zurück. Ebenso das Meereisvolumen. Dies ist zum Teil Folge der gegenwärtigen warmen Phase der langfristigen, natürlichen Atlantischen Multidekadischen Oszillation. Anthropogene Erwärmung und geänderte Windsysteme könnten den Rückgang des Meereises beschleunigen. Doch auch der Übergang der Atlantischen Multidekadischen Oszillation in eine kühlere Phase ist denkbar. Dann könnte sich das Meereis erholen. Sogar eine Umkehr des Erwärmungstrends ist denkbar. Der Rückgang des arktischen Meereises hat einen großen Einfluss auf atmosphärische und ozeanische Zirkulationssysteme. Die erwarteten Veränderungen würden auch das Klima in Europa betreffen.

\section{Uwe Jenisch}

\section{Arktis und Seerecht}

Seegrenzen, Festlandsockelansprüche und Verkehrsrechte

Die Arktis ist zum Politikum geworden. Die Arktisanrainer streben danach, ihre Nutzungsrechte räumlich und inhaltlich auszuweiten. Erweiterte Festlandsockel, komplizierte Seegrenzen, Schifffahrts- und Forschungsrechte sowie Umweltschutzpflichten richten sich jedoch im Interesse der Staatengemeinschaft nach den Regeln des UN-Seerechtsübereinkommens von 1982. Der Mechanismus für einen friedlichen Interessenausgleich ist vorhanden. Die Nicht-Anlieger-darunter 
die Staaten der EU - wie auch die Internationale Meeresbodenbehörde müssen ihre Rechte und Pflichten im Arktischen Ozean gegenüber den Regionalisierungsbestrebungen der Anrainer verteidigen.

\section{Elvira Pushkareva}

\section{Die Arktis im Völkerrecht}

Der starke Rückgang der sommerlichen Eisbedeckung im Nordpolarmeer hat eine völkerrechtliche Debatte über nationale Hoheitsechte im Arktischen Ozean entfacht. Es geht um Rohstoffförderung, Schifffahrt und Fischerei. Die grundsätzliche Frage lautet, ob das moderne Seerecht oder besondere Gewohnheitsrechte der Anrainerstaaten des Nordpolarmeers gelten. Auch wenn wahrscheinlich das Seerechtsübereinkommen angewendet werden wird, könnte es Russland und Kanada gelingen, unter Berufung auf historische Rechtstitel bei seiner Anwendung Sonderregeln durchzusetzen. Die Anerkennung »historischer Gewässer« würde die von den beiden Staaten bereits festgelegten Basislinien, von denen die im Seerechtsübereinkommen definierten Zonen bemessen werden, bestätigen.

\section{Karl Hinz}

Wem gehört die zentrale Arktis?

Geologie, Bathymetrie und das Seerecht

Eine Reihe von Anrainerstaaten des Nordpolarmeers erheben Ansprüche auf ein exklusives Recht zur Förderung von Rohstoffen in der zentralen Arktis. Das Seerechtsübereinkommen der Vereinten Nationen legt fest, welche Voraussetzungen erfüllt sein müssen, damit diese Ansprüche gerechtfertigt sind. Will der Küstenstaat jenseits von 200 Seemeilen souveräne Rechte über den sogenannten Festlandsockel ausüben, muss er mit bathymetrischen, geodätischen, geophysikalischen und geologische Daten nachweisen, dass sich die natürliche Verlängerung seines Landgebietes über diese juristisch festgelegte Grenze hinaus erstreckt. Russland ist mit einem solchen Antrag bei der UN-Kommission zur Begrenzung des Festlandsockels gescheitert, bereitet aber einen neuen Antrag mit einer verbesserten technischen Dokumentation vor.

\section{Thijs Duyzings}

Der Nördliche Seeweg

\section{Russlands Schifffahrtsregeln und das Völkerrecht}

Die sommerliche Eisbedeckung im Nordpolarmeer ist stark zurückgegangen. Schon hoffen Reedereien auf eine kürzere Route von Europa nach Asien. Doch ist umstritten, ob für die Schifffahrt auf dem Nördlichen Seeweg das internationale Seerecht oder nationales russländisches Recht anzuwenden ist. Der Status einiger Gewässer und Meerengen ist offen und Russlands Verwendung gerader Basislinien stößt auf Widerspruch. Konkurrierende Auffassungen gibt es auch zu der Frage, ob die im Seerechtsübereinkommen formulierten Ausnahmebestimmungen für eisbedeckte Gebiete die Anwendung nationaler Regeln für die Schifffahrt anstelle der Vorschriften des Seerechts erlaubt.

\section{Hilmar Rempel}

\section{Rohstoffe der Arktis}

\section{Potentiale und Vorkommen}

Die Arktis verfügt über ein bedeutendes Potential an Erdgas, Erdöl und anderen Rohstoffen. Die Entwicklung der Felder erfordert enorme Investitionen und technische Kompetenzen. Der im Zuge der Klimaänderung prognostizierte Eisrückgang könnte die Exploration und Gewinnung der Rohstoffe begünstigen. Allerdings sind dabei wegen des sensiblen Ökosystems höchste Umweltstandards anzuwenden. Der größte Teil der Vorkommen liegt in Gebieten, deren rechtlicher Status geklärt ist. Das Bild vom Konflikt um die Ressourcen ist übertrieben. Vielmehr bietet sich eine gemeinsame Erschließung der Ressourcen an.

\section{Indra Øverland}

Kooperation statt Konfrontation

Štokman, Jamal und Russlands Energiepolitik in der Arktis

Russlands Arktispolitik wird oft in geopolitischen Kategorien gedeutet. Doch im Hohen Norden findet kein von Energiehunger angetriebener Ressourcenwettlauf statt. Der Großteil der in der Arktis vermuteten Öl- und Gasvorkommen 
liegt in Gebieten, in denen Russland unumstritten das alleinige Recht zur Förderung hat. Zu beobachten ist daher eher ein Schönheitswettbewerb, bei dem internationale Ölkonzerne um die Beteiligung an den attraktivsten Erschließungsvorhaben konkurrieren. Als Modell könnte das Štokman-Projekt dienen. Verschiedene Entwicklungen auf den internationalen Öl- und Gasmärkten gefährden allerdings dieses Vorhaben.

\section{Stephen Fortescue}

Erze und OIigarchen

Die Bergbauindustrie in Russlands Nordwesten

Der Nordwesten Russlands ist reich an Bodenschätzen. Der Bergbau spielt daher insbesondere auf der Kola-Halbinsel ein erhebliche sozioökonomische Rolle. Die Geologie der Lagerstätten und die Geochemie der Erze erschweren allerdings den Abbau. Daher sind internationale Bergbauunternehmen kaum in der Region vertreten, und die vertikal integrierten russischen Kapitalgesellschaften der Grundstoffindustrie kämpfen mit zahlreichen Problemen. Aufgrund seiner großen wirtschaftlichen Bedeutung für den gesamten Nordwesten ist dem Bergbau jedoch die Aufmerksamkeit des Staates gewiss.

\section{Bettina Rudloff}

Fisch im Wasser?

Die EU und die Arktisfischerei

Die Arktis ist als Fischereigebiet derzeit nur von regionaler Bedeutung. Global gesehen haben die Fangmengen kaum Bedeutung, und auch für die Fischerei der EU-Staaten spielt die Arktis kaum eine Rolle. Wichtig ist die EU lediglich als Handelspartner einzelner arktischer Fischfangnationen. Trotz Überfischung sind Fangquoten- und Handelskonflikte allerdings selten. Welche Auswirkungen der Klimawandel auf zukünftige Fangpotentiale hat, ist unklar. Schon die Daten über aktuelle Fischbestände sind sehr unzuverlässig. Die Ökosysteme sind zu komplex und die wissenschaftlichen Daten zu lückenhaft, als dass sich Prognosen über die ökonomischen Potentiale des Fischfangs im Falle eines Rückgangs des Meereises aufstellen lassen würden.

\section{Karl Magnus Eger, Arnfinn Jørgensen-Dahl \\ Kurzer Weg, hohes Risiko}

Der Nördliche Seeweg: eine euro-asiatische Schiffsroute?

Die Nordostpassage wird seit einigen Jahren als neue Route für die kommerzielle Schifffahrt zwischen Europa und Asien gehandelt. Fahrten zwischen nordeuropäischen und nordpazifischen Häfen könnten so deutlich kürzer werden. Allerdings ist die Navigation trotz des Rückgangs der sommerlichen Eisbedeckung im Nordpolarmeer weiter schwierig. Im zentralen Abschnitt, dem von Russland kontrollierten Nördliche Seeweg, sind die Fahrten genehmigungspflichtig, Eisbrecherbegleitung ist obligatorisch. Zusammen mit hohen Versicherungskosten treibt dies die Transportkosten in die Höhe. Trotz der großen Treibstoff- und Zeiteinsparungen hängt es vom Einzelfall ab, ob die Nordroute tatsächlich günstiger ist als der Weg über den Suez-Kanal.

\section{Elena Nikitina}

Russlands dreckige Arktis

Umweltverschmutzung und ökologische Ansätze

Einige arktische Regionen Russlands sind ökologische Krisengebiete. In der Gegend um die Stadt Noril'sk sowie auf der Kola-Halbinsel verursacht der Bergbau eine massive Luft- und Wasserverschmutzung. Die Ökosysteme großer Gebiete sind zerstört. Katastrophal ist die Lage auch in den Öl- und Gasfördergebieten. Aus Pipelinelecks austretendes Öl verseucht Böden und Gewässer, das Abfackeln von Begleitgas verschmutzt die Luft, schweres Gerät führt zu Bodendegradation. In den letzten Jahren sind Ansätze eines ökologischen Bewusstseins entstanden. Moskau hat den Umweltschutz zu einem zentralen Thema seiner Arktispolitik erklärt. Nun müssen die zahlreichen Umweltstrategien auch umgesetzt werden. 


\section{Sandra Cavalieri et al.}

\section{Spurensuche}

\section{Der ökologische Fußabdruck der EU in der Arktis}

Die fragilen Ökosysteme der Arktis sind in Gefahr. Beim Bergbau und der Metallverhüttung werden in großen Mengen Ruß, Stickoxide und Schwefeldioxid freigesetzt. Toxische organische Schadstoffe und Schwermetalle reichern sich über die Nahrungskette in den Organismen von Tieren und Menschen an. Ein zusätzlicher Stressfaktor für die Ökosysteme ist der Klimawandel. Die biologische Vielfalt droht durch invasive Arten abzunehmen. Die Staaten der Europäischen Union tragen erheblich zur Umweltverschmutzung in der Nordpolregion bei. Ihr ökologischer Fußabdruck in der Arktis geht zum Großteil auf den Import von Waren und Dienstleistungen aus der Region zurück. Die EU sollte ihre Umweltschutzbemühungen in einer Umweltstrategie für die Arktis bündeln.

\section{Christoph Humrich, Klaus Dieter Wolf \\ Krieg in der Arktis?}

Konfliktszenarien auf dem Prüfstand

Als 2007 eine Expedition auf dem Meeresgrund des geographischen Nordpols Russlands Flagge absetzte, löste dies international Aufregung aus. Der Konflikt um den Festlandsockel schien zu eskalieren. Manche Beobachter malen Krisen und Kriege um die Rohstoffe der Arktis an die Wand. Wer die Fakten analysiert, kommt zu einem anderen Befund. Die Eskalation eines Konflikts um die Arktis ist unwahrscheinlich.

\section{Kristian Åtland}

\section{Im Norden nicht Neues?}

\section{Die Arktis in Russlands Sicherheitspolitik}

Die Sowjetunion betrachtete die Arktis als ein Gebiet von großer militärischer Bedeutung. Seit dem Ende des Ost-WestKonflikts hat sich dies grundlegend geändert. Die militärische Dimension spielt in Russlands Arktispolitik eine deutlich geringere Rolle. Moskau kooperiert über die ehemaligen Blockgrenzen hinweg mit anderen Staaten und beteiligt sich an zirkumpolaren regionalen Organisationen. Zudem spielt eine Reihe großer Konzerne heute ein gewichtige Rolle im Hohen Norden. Obwohl Gefahr für Russland viel eher an den südlichen Grenzen des Landes droht, wird die Arktis in Moskau immer noch als ein Raum angesehen, der nicht nur große wirtschaftliche Bedeutung hat, sondern auch sicherheitspolitisch relevant ist. Das Konfliktpotential in der Arktis sollte dennoch nicht überschätzt werden.

\section{Geir Hønneland}

\section{Kompromiss als Routine}

\section{Russisch-norwegische Konfliktlösung in der Barentssee}

Russland und Norwegen haben im April 2010 überraschend ihre Grenzstreitigkeiten in der Barentssee beigelegt. Ausschlaggebend für die Unterzeichnung eines Abkommens über die zukünftige Seegrenze war die in den vierzig Jahren zuvor gewonnene Verhandlungsroutine. Die Zusammenarbeit in der Fischereiwirtschaft und seit den späten 1980er Jahren auch im Umweltschutz förderte das Vertrauen zwischen den beiden Staaten. Der Kompromiss wurde selbst dann zum Normalfall, wenn die Positionen zuvor weit auseinandergelegen hatten.

\section{Klaus Gestwa}

Polarisierung der Sowjetgeschichte

\section{Die Antarktis im Kalten Krieg}

Als kultur- und geschichtsloser Raum erhielt die Antarktis erst in den 1950er Jahren ihren festen Platz auf der politischen Weltkarte. Überzogen mit einem dichten Netz von Forschungsstationen und geschützt durch ein internationales Vertragswerk ist der eisige sechste Kontinent zu einem einzigartigen globalen Raum mit normativem Vorbildcharakter geworden. Die Antarktis bot eine fabelhafte Projektionsfläche, auf der sich die »sozialistische Gemeinschaft« darstellen ließ. Am Südpol erschien der Ostblock im Kleinformat bald nicht mehr nur als gedachte, sondern als gelebte Gemeinschaft. 


\section{Michael Hamel-Green \\ Atomwaffenfreie Zone Arktis \\ Vorbilder und Perspektiven}

Die Arktis wurde im Ost-West-Konflikt zu einer der militarisiertesten Regionen der Welt. Im Nordpolarmeer kreuzten strategische U-Boote. Für nukleare Abschreckung sollten auch Langstreckenbomber und Interkontinentalraketen sorgen, deren Route über den Nordpol geführt hätte. Heute bietet sich die Chance auf Errichtung einer Atomwaffenfreien Zone in der Arktis. Ein Vorbild zur Entmilitarisierung der Arktis bietet der Antarktisvertrag. Die Einrichtung von Atomwaffenfreien Zonen in anderen Weltregionen hat gezeigt, dass die Initiative nicht von den Atomwaffenstaaten ausgehen muss. Wenn die Zivilgesellschaft Druck ausübt und die Staaten ohne Atomwaffen vorangehen, könnten die USA und Russland nachziehen.

\section{Kirsti Stuvøy, Brigt Dale}

Der Arktische Rat

Gesundheitsmanagement zwischen Wissenschaft und Politik

Der Arktische Rat ist die wichtigste Institution für das Regieren jenseits des Nationalstaates im Hohen Norden. Er trifft keine rechtsverbindlichen Entscheidungen, sondern beeinflusst Politik in der Arktis mit dem grundlegenden Wissen, das seine Arbeitsgruppen zur Verfügung stellen. Besonders erfolgreich ist sein Umweltmonitoring. Im Bereich des Gesundheitsmanagements, dem sich der Rat in den letzten Jahren zugewandt hat, zeigen sich aber auch die Grenzen der wissenschaftlichen Politikberatung. Letztlich muss der Rat doch politische Entscheidungen fällen, um als einflussreiche Institution in der Arktis Bestand zu haben.

\section{Vasilij Golovanov}

\section{Erde ohne Götter}

Kolguev liegt auf der »westabgewandten Seite der europäischen Geschichte«. Einst fegten auf der Insel in der Barentssee die Rentiernomaden der Nenzen mit Gänsefedern die Tundra, damit kein Stäubchen am Standplatz zurückbleibt. Die Sowjetunion hat einen Fundus des Weltalphabets zurückgelassen: Atommüll und Bierdosen, Fässer und Gasmasken, Holzteile und Hubschrauberverkleidung. Am Nordende des Kontinents ist das Zeitenende bereits gekommen.

\section{Ulrich Schmid}

\section{Eurasien oder Skandoslavien?}

Der »Norden« im kulturellen Selbstverständnis Russlands

Das publikumswirksame Aufpflanzen einer Nationalflagge aus Titan auf dem Meeresgrund unter dem Nordpol im Jahr 2007 hat deutlich gemacht, wie ernst Russlands geopolitische Ambitionen im Norden sind. Einige prominente kulturhistorische Diskurslinien belegen das Selbstverständnis Russlands als »nördliche« Nation. Oft wurde diese Dimension zugunsten der klischeehaften Gegenüberstellung Russland - Europa vernachlässigt. Doch auch die russischen Nordkonzeptionen sind keineswegs homogen. In den vergangenen zwei Jahrhunderten wurde der Norden immer wieder als Ursprungsort nationaler Kultur und Staatlichkeit imaginiert. Gleichzeitig gab es Absetz- und Gegenbewegungen, in denen das autochthon Slavische gegen das als fremd empfundene nördlich-skandinavische Element ausgespielt wurde.

\section{Florian Stammler, Elena Khlinovskaya}

Einmal »Erde« und zurück

\section{Bevölkerungsbewegung in Russlands Hohem Norden}

Im Vergleich zu anderen arktischen Regionen ist Russlands Arktis wesentlich dichter besiedelt. Dies ist das Ergebnis einer Bevölkerungspolitik, die in erster Linie der Industrialisierung diente. In der Sowjetunion waren Deportationen, durch Subventionen geförderte Umsiedlungen und Sesshaftmachung von Nomaden die Instrumente dieser Politik. Russland verfolgt heute andere politische und wirtschaftliche Ziele und versucht, unrentabel gewordene Siedlungen im Norden aufzulösen. Doch die Rücksiedlung stößt auf Widerstand. So leben in den neuen Geisterstädten des Nordens noch immer oder wieder einstige Umsiedler. 


\section{T. Heleniak, T. Holzlehner, E. Khlinovskaya}

\section{Der große Exodus}

Demographische Trends an Russlands nördlicher Peripherie

Das Ende der Sowjetunion ging mit dem Zusammenbruch der zentralen Verwaltungswirtschaft einher. Das löste eine massive Abwanderung der Menschen insbesondere aus den peripheren Regionen Russlands aus. Seit 1991 hat der Hohe Norden fast ein Fünftel seiner Bevölkerung verloren. Das Gebiet Magadan verließen 59 Prozent, den Autonomen Kreis der Tschuktschen gar drei Viertel aller Einwohner. Das hat gravierende Konsequenzen für die sozioökonomische Lage der Bleibenden sowie für die Infrastruktur, das Siedlungswesen und den Arbeitsmarkt.

\section{Johannes Rohr}

Anpassung und Selbstbehauptung

Die indigenen Völker in Russlands Hohem Norden

Russland erkennt 40 Völker des Hohen Nordens als indigen an. Ihnen gewährt der Staat besonderen Schutz. Gleichwohl bleiben diese Völker Bittsteller. Die Erdöl- und Erdgasförderung, der Bergbau sowie Staudammprojekte haben nach wie vor verheerende Auswirkungen auf ihren Lebensraum. Gegen große Industriekonzerne können sie sich nur erfolgreich zur Wehr setzen, wenn Russland die in internationalen Erklärungen formulierten Kollektivrechte indigener Völker anerkennt.

\section{Anna Stammler-Gossmann}

»Indigene Völker«

Historische Wurzeln der russländischen Minderheitenpolitik

Russland erkennt seit 1993 eine Reihe von Völkern als indigen an. Die staatliche Definition von Indigenität ist allerdings mit den in internationalen Erklärungen zu den Rechten indigener Völker formulierten Kriterien nur teilweise kompatibel. Dies hat mit tief verwurzelten Traditionen der zarischen und sowjetischen Nationalitätenpolitik zu tun.

\section{Christoph Humrich}

\section{Die Zukunft der Arktis}

\section{Ein Literaturbericht}

Die Arktis genießt große publizistische Aufmerksamkeit. Ursachen sind die Folgen des Klimawandels, der vermutete Rohstoffreichtum sowie das Echo auf Russlands Tauchfahrt zum Meeresgrund am Nordpol. Noch mangelt es an analytischen Grundlagen, um die vielfältigen Entwicklungen einzuordnen. Auch fehlen innovative Vorschläge für eine nachhaltige politische, sozioökonomische und ökologische Entwicklung der Region. 


\section{Abstracts aus Osteuropa 4/2011 mit Russlandbezug}

Osteuropa 4/2011 ist erschienen: Unter dem Titel »Amnesie International« geht es um den zweiten Prozess gegen Chodorkovskij und Lebedev, die Theorie der Erinnerung und die Praxis des Umgangs mit dem Stalinismus sowie Brechts Blick auf den Hitler-Stalin-Pakt sowie polnische Judaika. Das Heft hat 184 Seiten, kostet $10.00 €$ und ist zu bestellen über osteuropa@dgo-online.org

Im einzelnen enthält es folgende Beiträge mit Bezug auf Russland:

Otto Luchterhandt

Verhöhnung des Rechts

Der zweite Strafprozess gegen Chodorkovskij und Lebedev

Ausgerechnet zu einer Zeit, in der Präsident Medvedev als Staatsoberhaupt den »Rechtsnihilismus« im Lande geißelt und zu dessen Bekämpfung aufruft, wird von einem Moskauer Gericht gegen die JUKOS-Manager Michail Chodorkovskij und Platon Lebedev ein weiteres Strafurteil gefällt, welches das von 2005 an Willkür und Zynismus übertrifft. Dies ergibt eine detaillierte Analyse der Anklage, des Sachverhalts, des strafprozessualen Vorgehens sowie des Urteils. Im Unterschied zum ersten Prozess regt sich nun in Teilen der Elite Widerstand.

\section{Arsenij Roginskij \\ Erinnerung und Freiheit}

Die Stalinismus-Diskussion in der UdSSR und Russland

Seit dem »Tauwetter« ist der Umgang mit Stalin und dem Stalinismus ein Gradmesser für die aktuelle Politik. »AntiStalinisten" plädieren für Freiheit und Reformen, „Stalinisten« für Ordnung und den starken Staat. Die Geschichtspolitik des Putin-Regimes passt in dieses Modell. Der Rückgriff auf Stalin und den Sieg im Zweiten Weltkrieg sollte dazu dienen, Putins autoritäre Herrschaft zu legitimieren. Antidemokratische Politik ging mit der mythischen Verklärung der totalitären Vergangenheit einher. Doch der nationalstalinistische Geist droht sich gegen seine Schöpfer zu wenden. $\mathrm{Zu}$ dessen Abwehr sowie aus innen- und außenpolitischen Motiven bringen sich Medvedev und Putin nun als »Antistalinisten« in Position. Die sowjetische Geschichte ist erneut Schauplatz eines Kampfes um die Zukunft des Landes.

\section{Heinrich Olschowsky}

"Wo einst das südöstliche Polen war ..."

Bertolt Brecht und der Hitler-Stalin-Pakt

Anders als für das polnische nationale Gedächtnis war der Hitler-Stalin-Pakt für die linke deutsche Erinnerungskultur lange fast ein Tabu. Noch heute ist die Erinnerung daran schwierig. Einer der Gründe war die verbreitete Vorstellung, Antifaschismus und Antistalinismus seien unvereinbar. Deshalb äußerte sich auch Bertolt Brecht, der das Unrecht des Pakts durchaus sah, dazu nicht öffentlich. In seinem 1941 publizierten Gedicht Kinderkreuzzug 1939 dagegen spricht Brecht eine zwar poetische, aber deutliche Sprache. Die Brecht-Forschung hat den historischen Hintergrund des Gedichts ignoriert. 


\section{belarus-}

analysen

www.laender-analysen.de/belarus/

\section{NEU: BELARUS-ANALYSEN}

- Im Mai 2011 erscheint die erste Ausgabe der Belarus-Analysen.

- Diese Hintergrundanalysen sollen bei der Beurteilung aktueller Ereignisse und langfristiger Entwicklungen in Belarus behilflich sein. Sie werden von der Forschungsstelle Osteuropa an der Universität Bremen, der Deutschen Gesellschaft für Osteuropakunde, Berlin und dem Internationalen Bildungs- und Begegnungswerk, Dortmund, mit finanzieller Unterstützung des Ost-Ausschusses der Deutschen Wirtschaft herausgegeben. Ihr Anliegen ist es, das Wissen, über das die wissenschaftliche Forschung in reichem Maße verfügt, für Politik, Wirtschaft, Medien und interessierte Öffentlichkeit verfügbar zu machen.

- Jede Ausgabe bietet eine kurze Analyse zu einem aktuellen Thema der politischen, wirtschaftlichen oder sozialen Entwicklung des Landes. Ergänzt wird sie mit Tabellen und Grafiken. Zusätzlich gibt es regelmäßig Kommentare und Hintergrundinformationen zu aktuellen Ereignissen sowie eine Chronik mit den wichtigsten Geschehnissen der vergangenen zwei Monate. Die Belarus-Analysen orientieren sich damit am erfolgreichen Konzept der LänderAnalysen (www.laender-analysen.de).

- Die Belarus-Analysen werden ab Mai 2011 alle zwei Monate erscheinen und auf Anforderung kostenlos per E-Mail als pdf-Datei versandt.

- Für ein kostenloses Abonnement senden Sie eine E-Mail mit dem Stichwort "Abo BelarusAnalysen« in der Betreffzeile an fsopr@uni-bremen.de. Ein Archiv der kommenden Ausgaben sowie weitere Informationen finden Sie im Internet unter www.laender-analysen.de/belarus. 
Ost-Ausschuss der Deutschen Wirtschaft oa

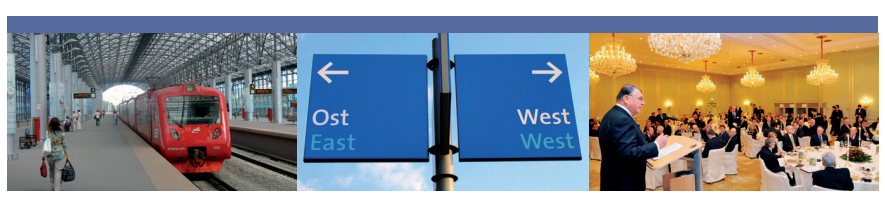

- Der Ost-Ausschuss der Deutschen Wirtschaft arbeitet seit 1952 aktiv daran, die Handelsund Investitionsbedingungen für deutsche Unternehmen in über 20 Ländern des östlichen Europas und Zentralasiens zu verbessern.

Unsere Konferenzen und Fachseminare informieren Sie über aktuelle Entwicklungen zwischen Zagreb und Wladiwostok.
Wir sind die Stimme der deutschen Wirtschaft in bilateralen Gremien und vernetzen Wirtschaft und Politik durch die Organisation exklusiver Wirtschaftsgespräche und Delegationsreisen.

Mit unseren Projekten fördern wir die marktwirtschaftliche Entwicklung in Osteuropa und investieren in die Aus- und Weiterbildung junger Menschen.

Weitere Informationen zu unseren Veranstaltungen, Publikationen und zur Mitgliedschaft im Ost-Ausschuss finden Sie unter: www.ost-ausschuss.de

\section{Brücke zu den Zukunftsmärkten}

\section{Ost-Ausschuss der Deutschen Wirtschaft}




\section{Kostenlose E-Mail-Dienste der Forschungsstelle Osteuropa und ihrer Partner auf www.laender-analysen.de}

\section{Belarus-Analysen}

Die Belarus-Analysen bieten einmal alle zwei Monate eine Kurzanalyse zu einem aktuellen Thema aus Politik, Wirtschaft oder Kultur, ergänzt um Grafiken und Tabellen. Zusätzlich gibt es eine Chronik aktueller Ereignisse.

Abonnement unter: fsopr@uni-bremen.de

\section{Caucasus Analytical Digest}

Der Caucasus Analytical Digest bietet einmal monatlich englischsprachige Kurzanalysen sowie illustrierende Daten zu einem aktuellen Thema. Zusätzlich gibt es eine Chronik aktueller Ereignisse.

Abonnement unter: http://www.res.ethz.ch/analysis/cad/

\section{Polen-Analysen}

Die Polen-Analysen bieten zweimal monatlich eine Kurzanalyse zu einem aktuellen Thema aus Politik, Wirtschaft oder Kultur, ergänzt um Grafiken und Tabellen. Zusätzlich gibt es eine Chronik aktueller Ereignisse.

Abonnement unter: http://www.deutsches-polen-institut.de/Newsletter/subscribe.php

\section{Russland-Analysen}

Die Russland-Analysen bieten vierzehntägig eine Kurzanalyse zu einem aktuellen Thema, ergänzt um Grafiken und Tabellen. Zusätzlich gibt es eine Chronik aktueller Ereignisse.

Abonnement unter: fsopr@uni-bremen.de

\section{Russian Analytical Digest}

Der Russian Analytical Digest bietet zweimal monatlich englischsprachige Kurzanalysen sowie illustrierende Daten zu einem aktuellen Thema.

Abonnement unter: http://www.res.ethz.ch/analysis/rad/

\section{Ukraine-Analysen}

Die Ukraine-Analysen bieten zweimal monatlich eine Kurzanalyse zu einem aktuellen Thema aus Politik, Wirtschaft oder Kultur, ergänzt um Grafiken und Tabellen. Zusätzlich gibt es eine Chronik aktueller Ereignisse.

Abonnement unter: fsopr@uni-bremen.de

\section{Zentralasien-Analysen}

Die Zentralasien-Analysen bieten monatlich eine Kurzanalyse zu einem aktuellen Thema aus Politik, Wirtschaft oder Kultur, ergänzt um Grafiken und Tabellen. Zusätzlich gibt es eine Chronik aktueller Ereignisse.

Abonnement unter: zentralasien-analysen@dgo-online.org

\section{Bibliographische Dienste}

Die vierteljährlich erscheinenden Bibliographien informieren über englisch- und deutschsprachige Neuerscheinungen zu Belarus, Polen, Russland, Tschechischer und Slowakischer Republik, Ukraine sowie zu den zentralasiatischen und kaukasischen Staaten. Erfasst werden jeweils die Themenbereiche Politik, Außenpolitik, Wirtschaft und Soziales. 